\title{
STRICHARTZ ESTIMATES AND THE NONLINEAR SCHRÖDINGER EQUATION ON MANIFOLDS WITH BOUNDARY
}

\author{
MATTHEW D. BLAIR, HART F. SMITH, AND CHRIS D. SOGGE
}

\begin{abstract}
We establish Strichartz estimates for the Schrödinger equation on Riemannian manifolds $(\Omega, \mathrm{g})$ with boundary, for both the compact case and the case that $\Omega$ is the exterior of a smooth, non-trapping obstacle in Euclidean space. The estimates for exterior domains are scale invariant; the range of Lebesgue exponents $(p, q)$ for which we obtain these estimates is smaller than the range known for Euclidean space, but includes the key $L_{t}^{4} L_{x}^{\infty}$ estimate, which we use to give a simple proof of well-posedness results for the energy critical Schrödinger equation in 3 dimensions. Our estimates on compact manifolds involve a loss of derivatives with respect to the scale invariant index. We use these to establish well-posedness for finite energy data of certain semilinear Schrödinger equations on general compact manifolds with boundary.
\end{abstract}

\section{INTRODUCTION}

Let $(\Omega, \mathrm{g})$ be a Riemannian manifold with boundary, of dimension $n \geq 2$, and let $v(t, x):[0, T] \times \Omega \rightarrow \mathbb{C}$ be the solution to the Schrödinger equation

$$
\left(i \partial_{t}+\Delta_{\mathrm{g}}\right) v(t, x)=0, \quad v(0, x)=f(x) .
$$

We assume in addition that $v$ satisfies either Dirichlet or Neumann boundary conditions

$$
\left.v(t, x)\right|_{\partial \Omega}=0 \quad \text { or }\left.\quad \partial_{\nu} v(t, x)\right|_{\partial \Omega}=0,
$$

where $\partial_{\nu}$ denotes the normal derivative along the boundary. In this work, we consider local in time Strichartz estimates for such solutions; these are a family of space-time integrability estimates of the form

$$
\|v\|_{L^{p}\left([0, T] ; L^{q}(\Omega)\right)} \leq C\|f\|_{H^{s}(\Omega)} .
$$

Here $H^{s}(\Omega)$ denotes the $L^{2}$ Sobolev space of order $s$, defined with respect to the spectral resolution of either the Dirichlet or Neumann Laplacian. The Lebesgue exponents will always be taken to satisfy $p, q \geq 2$, and always the Sobolev index satisfies $s \geq 0$.

The consideration of high frequency bump function solutions to (1) shows that $p, q, s$ must satisfy

$$
\frac{2}{p}+\frac{n}{q} \geq \frac{n}{2}-s
$$

The authors were supported by National Science Foundation grants DMS-0801211, DMS0654415, and DMS-0555162. 
In the case where equality holds in (3) the estimate is said to be scale invariant; otherwise, there is said to be a loss of derivatives in the estimate, as it deviates from the optimal regularity predicted by scale invariance.

Strichartz estimates are most well understood over Euclidean space, where $\Omega=$ $\mathbb{R}^{n}$ and $\mathrm{g}_{i j}=\delta_{i j}$. In this case, the scale invariant estimates hold with $s=0$ and $T=\infty$. See for example Strichartz [21], Ginibre and Velo [8], Keel and Tao [15], and references therein. Scale invariant estimates for $s>0$ then follow by Sobolev embedding; such estimates will be called subcritical, as their proof does not use the full rate of dispersion for the equation (1).

This paper is primarily concerned with proving scale invariant Strichartz estimates on the domain exterior to a non-trapping obstacle in $\mathbb{R}^{n}$, that is, $\Omega=\mathbb{R}^{n} \backslash \mathcal{K}$ for some compact set $\mathcal{K}$ with smooth boundary. Non-trapping means that every unit speed broken bicharacteristic escapes each compact subset of $\Omega$ in finite time. While we are only able to prove such estimates for a restricted range of subcritical $p, q$, we do obtain estimates with applications to wellposedness in the energy space for semilinear Schrödinger equations when $n=3$.

The key new step in this paper is to establish (for the same range of $p, q$ ) scale invariant estimates for the semi-classical Schrödinger equation on a general compact Riemannian manifold with boundary. The step from these local estimates to the case of exterior domains depends on the local smoothing bounds of Burq, Gérard, and Tzvetkov [4]. When $\mathcal{K}$ is assumed to be non-trapping, they proved that

$$
\|\psi v\|_{L^{2}\left([0, T] ; H^{s+\frac{1}{2}}(\Omega)\right)} \leq C\|f\|_{H^{s}(\Omega)}, \quad \psi \in C_{c}^{\infty}(\bar{\Omega}), \quad s \in[0,1] .
$$

This inequality is a natural formulation of the local smoothing estimates for Euclidean space which originated in the work of Constantin and Saut [6], Sjölin [18], and Vega [23]. The estimate (4) was used in [4] to obtain Strichartz estimates with a loss of $1 / p$ derivatives, by combining the gain in regularity in (4) with Sobolev embedding, in order to prove space-time integrability estimates near the obstacle.

Improved results were obtained by Anton [1], which show that Strichartz estimates hold with a loss of $\frac{1}{2 p}$ derivatives. The approach in [1] combines the local smoothing estimates (4) with a semi-classical parametrix construction, rather than Sobolev embedding. We remark that further improvement is possible by using the parametrix construction of the authors in [2], to yield a loss of $\frac{1}{3 p}$ derivatives. This is currently the best known estimate for critical $p, q$, that is, $\frac{2}{p}+\frac{n}{q}=\frac{n}{2}$, except for the case where $\mathcal{K}$ is strictly convex. For the exterior domain to a strictly convex $\mathcal{K}$, the full range of Strichartz estimates (except for endpoints) was obtained by Ivanovici [12] for Dirichlet conditions, using the Melrose-Taylor parametrix construction.

The use of local smoothing to establish Strichartz bounds has origins in the work of Journé, Soffer, and Sogge [14], and of Staffilani and Tataru [19]. Both deal with perturbations of the flat Laplacian in $\mathbb{R}^{n}$, and establish estimates with no loss of derivatives. The paper [14] considered the case of potential terms $-\Delta+V$, whereas [19] considers non-trapping metric perturbations. In both cases, one has local smoothing estimates similar to (4).

More recently, Planchon and Vega [17] used a bilinear virial identity to obtain the scale invariant estimate (2) where $p=q=4, s=\frac{1}{4}$ in $n=3$ dimensions (along with a range of related inequalities), for the Dirichlet problem on non-trapping exterior domains. These estimates were applied to semilinear Schrödinger equations, 
showing that for defocusing, energy subcritical nonlinearities, one has global existence for initial data in $H^{1}(\Omega)$. For strictly convex $\mathcal{K}$, the work [12] establishes global existence for the energy critical semilinear equation, focusing or defocusing, for small Dirichlet data in $H^{1}(\Omega)$.

In the present work, we establish the Strichartz estimates (2) for a range of subcritical $p, q$. The key tool is a microlocal parametrix construction previously used for the wave equation in [20] and [3]. This approach treats both Dirichlet and Neumann boundary conditions, and applies to general non-trapping obstacles.

Theorem 1.1. Let $\Omega=\mathbb{R}^{n} \backslash \mathcal{K}$ be the exterior domain to a compact non-trapping obstacle with smooth boundary, and $\Delta$ the standard Laplace operator on $\Omega$, subject to either Dirichlet or Neumann conditions. Suppose that $p>2$ and $q<\infty$ satisfy

$$
\begin{cases}\frac{3}{p}+\frac{2}{q} \leq 1, & n=2, \\ \frac{1}{p}+\frac{1}{q} \leq \frac{1}{2}, & n \geq 3 .\end{cases}
$$

Then for the solution $v=\exp (i t \Delta) f$ to the Schrödinger equation (1), the following estimates hold

provided that

$$
\|v\|_{L^{p}\left([0, T] ; L^{q}(\Omega)\right)} \leq C\|f\|_{H^{s}(\Omega)},
$$

$$
\frac{2}{p}+\frac{n}{q}=\frac{n}{2}-s
$$

For Dirichlet boundary conditions, the estimates hold with $T=\infty$.

That one may take $T=\infty$ in (6) for Dirichlet boundary conditions is a consequence of the fact that (4) holds for $T=\infty$ in the Dirichlet case.

We now consider estimates for compact Riemannian manifolds $\Omega$, with $\Delta_{\mathrm{g}}$ the Laplace-Beltrami operator for g. Burq, Gérard, and Tzvetkov showed in [5] that for $p>2$ estimates hold with a loss of $\frac{1}{p}$ derivatives in case $\partial \Omega=\emptyset$. The same result was established for compact manifolds with geodesically concave boundary in [12]. For general boundaries, we establish estimates with the same loss of $\frac{1}{p}$ derivatives, valid for $(p, q)$ satisfying $(5)$. For such $(p, q)$, this is an improvement over the estimates of [2], which involve a loss of $\frac{4}{3 p}$ derivatives.

Theorem 1.2. Let $\Omega$ be a compact Riemannian manifold with boundary. Suppose that $p>2$ and $q<\infty$ satisfy (5). Then for the solution $v=\exp \left(i t \Delta_{\mathrm{g}}\right) f$ to the Schrödinger equation (1), the following estimates hold for fixed finite $T$

$$
\|v\|_{L^{p}\left([0, T] ; L^{q}(\Omega)\right)} \leq C\|f\|_{H^{s+\frac{1}{p}}(\Omega)}
$$

for $p, q, s$ satisfying (7).

As with [5] and [12, Corollary 1.5], the loss of $\frac{1}{p}$ arises as a consequence of using a representation for solutions that is valid only in a local coordinate chart; that is, on a semi-classical time scale.

In the last two sections of this paper we present applications of the above theorems to well-posedness of semilinear Schrödinger equations in three space dimensions with finite energy data. In Section 5, we use Theorem 1.1 and interpolation to establish the $L_{t}^{4} L_{x}^{\infty}$ Strichartz estimate. This estimate yields a simple proof of well-posedness for small energy data to the energy critical equation on exterior domains, a result first established by Ivanovici and Planchon [13]. In Section 6, we establish a variant in three dimensions of Theorem 1.2 for the case $p=2$, for data 
$u$ localized to dyadic frequency scale $\lambda$. The estimate involves a loss of $(\log \lambda)^{2}$ relative to the estimates of [5]. Following the Yudovitch argument as in [5, Section 3.3], we use this to establish well-posedness for finite energy data to certain semilinear Schrödinger equations, on general three dimensional compact manifolds with boundary. The logarithmic loss in the estimates restricts our result to slower growth nonlinearities than considered in [5] for manifolds without boundary. For particular three dimensional manifolds without boundary, recent results have been obtained for the energy critical case by Herr [9], Herr, Tataru, and Tzvetkov [10], and Ionescu and Pausader [11].

The outline of this paper is as follows. In section 2, we reduce Theorems 1.1-1.2 to estimates on the unit scale within a single coordinate chart. Section 3 outlines the angular localization approach from [20], and introduces a wave packet parametrix construction. Estimates on the parametrix are then developed in section 4. We conclude in sections 5 and 6 with the applications to semilinear Schrödinger equations.

Throughout this paper we use the following notation. The expression $X \lesssim Y$ means that $X \leq C Y$ for some $C$ depending only on the manifold, metric, and possibly the triple $(p, q, s)$ under consideration. Also, we abbreviate $L^{p}\left(I ; L^{q}(U)\right)$ by $L^{p} L^{q}(I \times U)$ or by $L_{T}^{p} L^{q}(U)$ when $I=[0, T]$. If $U=\mathbb{R}^{n}$ we write $L_{T}^{p} L^{q}$. As will be seen below, the last component of an $n$-vector will take on special meaning, hence we will often write $x=\left(x^{\prime}, x_{n}\right)$ so that $x^{\prime}$ denotes the first $n-1$ components.

We conclude this introduction with a remark on the Sobolev spaces that we use in the case of exterior domains. In the above theorems, the Sobolev space $H^{s}(\Omega)$ and the operator $\exp (i t \Delta)$ are defined using the spectral resolution of $\Delta$ subject to the chosen Dirichlet or Neumann boundary condition $B$; in particular, the linear evolution preserves $H^{s}(\Omega)$. The space $H^{2}(\Omega)$ is then equal to the subspace of $H^{2}(\bar{\Omega})$ satisfying $B u=0$, and for $0 \leq s \leq 2$, the space $H^{s}(\Omega)$ can be defined by interpolation. For $s \geq 2$, these spaces satisfy $u \in H^{s}(\Omega)$ if and only if $B u=0$ and $\Delta u \in H^{s-2}(\Omega)$. Thus, for large values of $s$, a function in $H^{s}(\Omega)$ satisfies the linear compatibility conditions $B\left(\Delta^{k} u\right)=0$, for $k$ for which this is defined. These compatibility conditions are necessary to bootstrap the local smoothing estimates (4) to higher orders $s$, as well as to insure $v(t, \cdot) \in H^{s}(\Omega)$, which is required to handle commutator terms with cutoff functions. We will also use that

$$
\|v\|_{H^{s}(\Omega)} \approx\|\psi v\|_{H^{s}(\tilde{\Omega})}+\|(1-\psi) v\|_{H^{s}\left(\mathbb{R}^{n}\right)},
$$

where $\psi \in C_{c}^{\infty}(\bar{\Omega})$ is such that $1-\psi$ vanishes on a neighborhood of $\partial \Omega$, and $\tilde{\Omega}$ is a compact manifold with boundary in which $\Omega \cap \operatorname{supp}(\psi)$ embeds isometrically; for example $\tilde{\Omega}=\Omega \cap[-R, R]^{n}$ with periodic boundary conditions and $R$ sufficiently large.

We use $H^{s}(\bar{\Omega})$ to denote the space of extendable elements, with no boundary conditions. For $\Omega$ an exterior domain, $H^{s}(\bar{\Omega})$ consists of restrictions of functions in $H^{s}\left(\mathbb{R}^{n}\right)$ to $\Omega$ with the quotient norm (minimal norm of an extension); for $\Omega$ compact we embed $\Omega$ in a compact manifold $\Omega^{\prime}$ without boundary, and $H^{s}(\bar{\Omega})$ consists of restrictions of elements $H^{s}\left(\Omega^{\prime}\right)$. By elliptic regularity, $H^{s}(\Omega) \subset H^{s}(\bar{\Omega})$. 


\section{Preliminary Reductions}

In this section, we reduce the inequalities in Theorems 1.1 and 1.2 to estimates on solutions to a pseudodifferential equation defined in a coordinate chart near the boundary. We start by considering the case of Theorem 1.1.

For $\Omega=\mathbb{R}^{n} \backslash \mathcal{K}$, we take $\psi \in C_{c}^{\infty}(\bar{\Omega})$ such that $1-\psi$ vanishes on a neighborhood of $\partial \Omega=\partial \mathcal{K}$. Then $v_{0}=(1-\psi) v$ satisfies the inhomogeneous Schrödinger equation on $\mathbb{R}^{n}$ :

$$
\left(i \partial_{t}+\Delta\right) v_{0}=[\psi, \Delta] v,\left.\quad v_{0}\right|_{t=0}=(1-\psi) f .
$$

Here, $(1-\psi) f \in H^{s}\left(\mathbb{R}^{n}\right)$, and by (4) we have $[\Delta, \psi] v \in L_{T}^{2} H^{s-\frac{1}{2}}\left(\mathbb{R}^{n}\right)$. (Although stated only for $s \in[0,1]$ in [4], it is easy to see by a bootstrap argument and interpolation that (4) holds for all $s \geq 0$, where $H^{s}$ is the is the intrinsic Sobolev space for the Dirichlet/Neumann conditions as above.) The Strichartz estimates for $v_{0}$ then follow from Proposition 2.10 of [4] together with Sobolev embedding. While [4] considers the case $s \in[0,1]$, the result of Proposition 2.10 of [4] follows for all $s>0$, since the free Schrödinger propagator $\exp (i t \Delta)$ on $\mathbb{R}^{n}$ commutes with differentiation.

We are thus reduced to establishing estimates on the term $\psi v$. We isometrically embed a neighborhood of $\operatorname{supp}(\psi)$ into a compact manifold $(\tilde{\Omega}, \mathrm{g})$ with boundary, where $\partial \tilde{\Omega}=\partial \Omega$. Then $v_{1}=\psi v$ satisfies the inhomogeneous Schrödinger equation on $\tilde{\Omega}$ :

$$
\left(i \partial_{t}+\Delta_{\mathrm{g}}\right) v_{1}=[\Delta, \psi] v,\left.\quad v_{1}\right|_{t=0}=\psi f .
$$

By (4), we are reduced to establishing the following estimate over a compact manifold with boundary $\tilde{\Omega}$,

$$
\|v\|_{L_{T}^{p} L^{q}(\tilde{\Omega})} \lesssim\|v\|_{L_{T}^{2} H^{s+\frac{1}{2}(\tilde{\Omega})}}+\left\|\left(i \partial_{t}+\Delta_{\mathrm{g}}\right) v\right\|_{L_{T}^{2} H^{s-\frac{1}{2}}(\tilde{\Omega})} .
$$

Here we use that $[\Delta, \psi]$ vanishes near $\partial \Omega$, hence maps $H^{s+\frac{1}{2}}(\tilde{\Omega}) \rightarrow H^{s-\frac{1}{2}}(\tilde{\Omega})$.

We next take a Littlewood-Paley decomposition of $v$ in the $x$ variable with respect to the spectrum for $\Delta_{\mathrm{g}}$. Precisely, we write

$$
v=\beta_{0}\left(-\Delta_{\mathrm{g}}\right)+\sum_{j=1}^{\infty} \beta\left(2^{-2 j}\left(-\Delta_{\mathrm{g}}\right)\right) v \equiv \sum_{j=0}^{\infty} v_{j},
$$

where $\sum_{j=1}^{\infty} \beta\left(2^{-2 j} s\right)=1$ for $s \geq 2$, and $\beta$ is supported by $s \in\left[\frac{1}{2}, 2\right]$. The low frequency terms are easily dealt with by Sobolev embedding, since the right hand side of (8) controls the $L_{T}^{p} H^{s-\frac{1}{2}}$ norm of $v$ for $2 \leq p \leq \infty$. The following square function estimate holds, for example by heat kernel methods,

$$
\|v\|_{L_{T}^{p} L^{q}(\tilde{\Omega})} \approx\left\|\left(\sum_{j}\left|v_{j}\right|^{2}\right)^{\frac{1}{2}}\right\|_{L_{T}^{p} L^{q}(\tilde{\Omega})} \leq\left(\sum_{j}\left\|v_{j}\right\|_{L_{T}^{p} L^{q}(\tilde{\Omega})}^{2}\right)^{\frac{1}{2}},
$$

where we use $p, q \geq 2$ in the last step. By orthogonality, the desired estimate (8) would then follow as a consequence of the following estimate,

$$
\left\|v_{j}\right\|_{L_{T}^{p} L^{q}(\tilde{\Omega})} \lesssim 2^{j\left(s+\frac{1}{2}\right)}\left\|v_{j}\right\|_{L_{T}^{2} L^{2}(\tilde{\Omega})}+2^{j\left(s-\frac{1}{2}\right)}\left\|\left(i \partial_{t}+\Delta_{\mathrm{g}}\right) v_{j}\right\|_{L_{T}^{2} L^{2}(\tilde{\Omega})} .
$$

Finally, we divide $[0, T]$ into intervals of length $2^{-j}$ and note that, since $p, q \geq 2$, by the Minkowski inequality it suffices to prove the above on each subinterval; that is, for $T=2^{-j}$. To summarize, Theorem 1.1 is thus reduced to establishing the following semiclassical result. 
Theorem 2.1. Let $\Omega$ be a compact Riemannian manifold with boundary, and $\Delta_{\mathrm{g}}$ the Laplace-Beltrami operator, subject to either Dirichlet or Neumann boundary conditions. Suppose that $p>2$ and $q<\infty$ satisfy (5) and (7).

Suppose also that, for all $t, v_{\lambda}(t, \cdot)$ is spectrally localized for $-\Delta_{\mathrm{g}}$ to the range $\left[\frac{1}{4} \lambda^{2}, 4 \lambda^{2}\right]$. Then the following estimate holds, uniformly over $\lambda$,

$$
\left\|v_{\lambda}\right\|_{L_{\lambda-1}^{p} L^{q}(\Omega)} \lesssim \lambda^{s}\left(\lambda^{\frac{1}{2}}\left\|v_{\lambda}\right\|_{L_{\lambda}^{2} L^{2}(\Omega)}+\lambda^{-\frac{1}{2}}\left\|\left(i \partial_{t}+\Delta_{\mathrm{g}}\right) v_{\lambda}\right\|_{L_{\lambda^{-1}}^{2} L^{2}(\Omega)}\right) .
$$

We observe that Theorem 1.2 also follows as a consequence of (10). To see this, we divide $[0, T]$ into subintervals of length $\lambda^{-1}$, and note that for $v_{\lambda}=\exp \left(i t \Delta_{\mathrm{g}}\right) f_{\lambda}$, on each subinterval the right hand side of (10) is bounded by $\lambda^{s}\left\|f_{\lambda}\right\|_{L^{2}(\Omega)}$. Summing the $L^{p}$ norm over a total of $\approx \lambda$ subintervals leads to

$$
\left\|v_{\lambda}\right\|_{L_{T}^{p} L^{q}(\Omega)} \lesssim \lambda^{s+\frac{1}{p}}\left\|f_{\lambda}\right\|_{L^{2}(\Omega)} \approx\left\|f_{\lambda}\right\|_{H^{s+\frac{1}{p}}(\Omega)} .
$$

Applying the square function estimate (9) as above yields Theorem 1.2.

We will establish (10) by the methods developed in [20] and [3] to obtain dispersive estimates for the wave equation on manifolds with boundary. We start by taking a finite partition of unity over $\Omega$, subordinate to a cover by coordinate patches. We restrict attention to a coordinate patch centered on $\partial \Omega$; the interior terms can be handled by the methods of [5], or by the parametrix construction of this paper. Thus, let $\psi \in C_{c}^{\infty}(\bar{\Omega})$ be supported in a boundary normal coordinate patch along $\partial \Omega$. The function $\psi v_{\lambda}$ is not sharply spectrally localized, but does remain spectrally concentrated in frequencies $\leq \lambda$. Precisely, for all $k \geq 0$,

$$
\left\|\psi v_{\lambda}\right\|_{L_{\lambda-1}^{2} H^{k}(\bar{\Omega})} \lesssim\left\|v_{\lambda}\right\|_{L_{\lambda-1}^{2} H^{k}(\Omega)} \lesssim \lambda^{k}\left\|v_{\lambda}\right\|_{L_{\lambda-1}^{2} L^{2}(\Omega)},
$$

and the same holds with $v_{\lambda}$ replaced by $\left(i \partial_{t}+\Delta_{\mathrm{g}}\right) v_{\lambda}$.

Letting $x_{n}$ denote geodesic distance to the boundary, and $x^{\prime}$ coordinates on $\partial \Omega$, in boundary normal coordinates the Laplace operator takes the form

$$
\Delta_{g} v=\rho^{-1} \sum_{1 \leq i, j \leq n} \partial_{i}\left(\mathrm{~g}^{i j} \rho \partial_{j} v\right)
$$

where $\rho=\sqrt{\operatorname{det} g_{l k}}$ and $\mathrm{g}^{i j}$ denotes the inverse of the metric $\mathrm{g}_{l k}$. Furthermore, $\mathrm{g}^{i n}=\mathrm{g}^{n i}=\delta_{i n}$, so there are no mixed $\partial_{x^{\prime}} \partial_{x_{n}}$ terms.

We now extend the metric $\mathrm{g}\left(x^{\prime}, x_{n}\right)$ in an even manner across $x_{n}=0$; the new metric $\mathrm{g}\left(x^{\prime},\left|x_{n}\right|\right)$, which we also denote by $\mathrm{g}$, is defined on an open subset of $\mathbb{R}^{n}$, and is of Lipschitz regularity. We extend the solution $\psi v_{\lambda}$ in an odd or even fashion, corresponding to Dirichlet or Neumann boundary conditions, to obtain a $C^{1,1}$ function. We will assume $\psi$ is chosen so that $\psi\left(x^{\prime}, x_{n}\right)$ is independent of $x_{n}$ near $x_{n}=0$. Since the extended Laplace operator is even, the regularity of $g$ and $v_{\lambda}$ show that the extended solution satisfies the extended equation across $x_{n}=0$,

$$
\left(i \partial_{t}+\Delta_{\mathrm{g}}\right)\left(\psi v_{\lambda}\right)=\left[\Delta_{\mathrm{g}}, \psi\right] v_{\lambda}+\psi\left(i \partial_{t}+\Delta_{\mathrm{g}}\right) v_{\lambda},
$$

where $\Delta_{\mathrm{g}} v_{\lambda}$ is extended oddly/evenly as is $v_{\lambda}$, and $\psi$ is even.

By choosing sufficiently small coordinate patches, and rescaling if necessary, we may assume that $g$ extends to all of $\mathbb{R}^{n}$, such that

$$
\left\|\mathrm{g}^{i j}-\delta^{i j}\right\|_{C^{0,1}\left(\mathbb{R}^{n}\right)} \leq c_{0} \ll 1, \quad \mathrm{~g}^{i j}=\delta^{i j} \text { if }|x|>1 .
$$

The odd (respectively even) extension operator maps functions in $H^{r}\left(\overline{\mathbb{R}_{+}^{n}}\right)$ satisfying $f\left(x^{\prime}, 0\right)=0$ (respectively $\partial_{x_{n}} f\left(x^{\prime}, 0\right)=0$ ) to functions in $H^{r}\left(\mathbb{R}^{n}\right)$, provided 
$r \in\left[0, \frac{5}{2}\right)$. The extension also commutes with differentiation in the $x^{\prime}$ variables. We observe that multiplication by functions such as g or $\rho$ preserves $H^{r}\left(\mathbb{R}^{n}\right)$ for $r \in\left[0, \frac{3}{2}\right)$, and multiplication by $\partial_{x} \rho$ preserves $H^{r}\left(\mathbb{R}^{n}\right)$ for $r \in\left[0, \frac{1}{2}\right)$. This can be seen, e.g., from the fact that $\langle\xi\rangle^{\frac{1}{2}-\varepsilon}$ is an $A_{2}$ weight in one dimension, and that $\partial_{x} \rho$ is a Calderón-Zygmund type multiplier in $x_{n}$.

It follows that the bound (11) holds to a limited extent for the extension of $\psi v_{\lambda}$ to $\mathbb{R}^{n}$. To quantify this, we introduce the following family of norms, for $r \geq 0$,

$$
\|f\|_{H^{r, \lambda}}=\sum_{|\alpha| \leq N}\left(\lambda^{-|\alpha|}\left\|\partial_{x^{\prime}}^{\alpha} f\right\|_{L^{2}\left(\mathbb{R}^{n}\right)}+\lambda^{-|\alpha|-r}\left\|\partial_{x^{\prime}}^{\alpha} f\right\|_{H^{r}\left(\mathbb{R}^{n}\right)}\right),
$$

and observe that $\|f\|_{H^{\sigma, \lambda}} \lesssim\|f\|_{H^{r, \lambda}}$ if $0 \leq \sigma \leq r$. Here $N$ is taken to be a fixed but sufficiently large number, which we allow to change in a given inequality. However, for the results of this paper $N$ need never exceed $n+2$.

By (11) and the above, it holds that for $2 \leq r<\frac{5}{2}$

$$
\begin{aligned}
\lambda^{\frac{1}{2}}\left\|\psi v_{\lambda}\right\|_{L_{\lambda-1}^{2} H^{r, \lambda}\left(\mathbb{R}^{n}\right)} & +\lambda^{-\frac{1}{2}}\left\|\left(i \partial_{t}+\Delta_{\mathrm{g}}\right)\left(\psi v_{\lambda}\right)\right\|_{L_{\lambda-1}^{2} H^{r-2, \lambda}\left(\mathbb{R}^{n}\right)} \\
& \lesssim \lambda^{\frac{1}{2}}\left\|v_{\lambda}\right\|_{L_{\lambda-1}^{2} L^{2}(\Omega)}+\lambda^{-\frac{1}{2}}\left\|\left(i \partial_{t}+\Delta_{\mathrm{g}}\right) v_{\lambda}\right\|_{L_{\lambda-1}^{2} L^{2}(\Omega)} .
\end{aligned}
$$

This bound also holds if we replace $\Delta_{\mathrm{g}}$ on the left side by the divergence form operator $\partial_{i} \mathrm{~g}^{i j} \partial_{j}$, since the difference $\rho^{-1}\left(\partial_{i} \rho\right) \mathrm{g}^{i j} \partial_{j}$ maps $H^{r, \lambda} \rightarrow H^{r-2, \lambda}$ with norm $\lambda$, provided $r \in\left[2, \frac{5}{2}\right)$. Since subsequent estimates will be only in terms of the left hand side of (12), we may thus set $\rho \equiv 1$, and replace $\Delta_{\mathrm{g}}$ by $\sum_{i j} \partial_{i} \mathrm{~g}^{i j} \partial_{j}$.

We next reduce matters to considering solutions that are strictly frequency localized on $\mathbb{R}^{n}$, and which satisfy an equation with frequency localized coefficients. For each $\mu$, we form regularized coefficients $\mathrm{g}_{\mu}^{i j}$ by truncating the Fourier transform of the $\mathrm{g}^{i j}$ so that

$$
\operatorname{supp}\left(\widehat{\mathrm{g}_{\mu}^{i j}}\right) \subset\{|\xi| \leq c \mu\},
$$

for some small constant $c$. We observe the following estimates

$$
\left\|\mathrm{g}_{\mu}^{i j}-\mathrm{g}^{i j}\right\|_{L^{\infty}\left(\mathbb{R}^{n}\right)} \lesssim \mu^{-1}, \quad\left\|\partial_{x}^{\alpha} \mathrm{g}_{\mu}^{i j}\right\|_{L^{\infty}\left(\mathbb{R}^{n}\right)} \lesssim \mu^{|\alpha|-1}, \quad|\alpha| \geq 1 .
$$

With slight abuse of notation we now set

$$
\Delta_{\mathrm{g}_{\mu}} v=\sum_{1 \leq i, j \leq n} \partial_{i}\left(\mathrm{~g}_{\mu}^{i j} \partial_{j} v\right) .
$$

We will prove in the next section the following estimate for $u_{\mu}(t, x)$ defined on $\left[0, \mu^{-1}\right] \times \mathbb{R}^{n}$, which are localized to spatial frequencies $\approx \mu$.

Lemma 2.2. Suppose that $(p, q, s)$ are as in Theorem 1.1, and $\widehat{u}_{\mu}(t, \xi)$ is supported in the region $\frac{1}{2} \mu \leq|\xi| \leq \frac{5}{2} \mu$. Then

$$
\left\|u_{\mu}\right\|_{L_{\mu-1}^{p} L^{q}} \lesssim \mu^{s+\frac{1}{2}}\left\|u_{\mu}\right\|_{L_{\mu^{-1}}^{2} L^{2}}+\mu^{s}\left\|\left(i \partial_{t}+\Delta_{\mathrm{g}_{\mu}}\right) u_{\mu}\right\|_{L_{\mu^{-1}}^{1} L^{2}} .
$$

Furthermore, if $\widehat{u}_{\mu}(t, \xi)$ is in addition localized to $\left|\xi^{\prime}\right| \leq \frac{3}{2}\left|\xi_{n}\right|$, then (15) holds for $p>2$ and $q<\infty$ satisfying (7) with $s \geq 0$; that is, without the restriction (5).

In the remainder of this section we reduce (10), and hence Theorems 1.1 and 1.2, to establishing Lemma 2.2 .

We start by considering the frequency components $\mu \leq \lambda$ of $\psi v_{\lambda}$ (by which we understand its odd/even extension to $\mathbb{R}^{n}$ ). Let $\beta_{\mu}(D)$ denote a Littlewood-Paley 
localization operator on $\mathbb{R}^{n}$ to frequencies $\approx \mu$, and consider $u_{\mu}=\beta_{\mu}(D)\left(\psi v_{\lambda}\right)$. For $\mu \leq \lambda,(15)$ and the Schwarz inequality imply

$$
\left\|u_{\mu}\right\|_{L_{\lambda^{-1}}^{p} L^{q}} \lesssim \mu^{s}\left(\lambda^{\frac{1}{2}}\left\|u_{\mu}\right\|_{L_{\lambda^{-1}}^{2} L^{2}}+\lambda^{-\frac{1}{2}}\left\|\left(i \partial_{t}+\Delta_{\mathrm{g}_{\mu}}\right) u_{\mu}\right\|_{L_{\lambda-1}^{2} L^{2}}\right) .
$$

Since $s \geq 0$ in our bounds, we may sum over dyadic values of $\mu \leq \lambda$ to establish (10) for the cutoff of $\psi v_{\lambda}$ to frequencies $\leq \lambda$, provided we bound the $\ell^{2}$ norm over $\mu$ of the terms in parentheses in (16) by the terms in parentheses in (10). By (12), this is a special case of the following estimate, which we establish for all $r \in\left[2, \frac{5}{2}\right)$,

$$
\begin{gathered}
\left(\sum_{\mu} \lambda\left\|u_{\mu}\right\|_{L_{\lambda-1}^{2} H^{r, \lambda}}^{2}+\lambda^{-1}\left\|\left(i \partial_{t}+\Delta_{\mathrm{g}_{\mu}}\right) u_{\mu}\right\|_{L_{\lambda-1}^{2} H^{r-2, \lambda}}^{2}\right)^{\frac{1}{2}} \\
\lesssim \lambda^{\frac{1}{2}}\left\|\psi v_{\lambda}\right\|_{L_{\lambda-1}^{2} H^{r, \lambda}}+\lambda^{-\frac{1}{2}}\left\|\left(i \partial_{t}+\Delta_{\mathrm{g}}\right)\left(\psi v_{\lambda}\right)\right\|_{L_{\lambda-1}^{2} H^{r-2, \lambda}} .
\end{gathered}
$$

Since $\beta_{\mu}$ is $L^{2}$ bounded and commutes with differentiation, this will follow from showing the fixed time estimate

$$
\left(\sum_{\mu}\left\|\left(\beta_{\mu} \Delta_{\mathrm{g}}-\Delta_{\mathrm{g}_{\mu}} \beta_{\mu}\right)\left(\psi v_{\lambda}\right)\right\|_{H^{r-2, \lambda}}^{2}\right)^{\frac{1}{2}} \lesssim \lambda\left\|\psi v_{\lambda}\right\|_{H^{r-1, \lambda}} .
$$

In this estimate we may replace $\Delta_{\mathrm{g}}=\partial_{i} \mathrm{~g}^{i j} \partial_{j}$ by $\mathrm{g}^{i j} \partial_{i} \partial_{j}$, and similarly for $\Delta_{\mathrm{g}_{\mu}}$. This follows since the difference $\left(\partial_{i} \mathrm{~g}^{i j}\right) \partial_{j}$ maps $H^{r-1, \lambda} \rightarrow H^{r-2, \lambda}$ with norm $\lambda$. By the Coifman-Meyer commutator estimate (see [22, Prop 4.1D]), for $\sigma \in[0, r-2]$,

$$
\left(\sum_{\mu}\left\|\left[\beta_{\mu}, \mathrm{g}\right] \partial_{x}^{2}\left(\psi v_{\lambda}\right)\right\|_{H^{\sigma}}^{2}\right)^{\frac{1}{2}} \lesssim\left\|\psi v_{\lambda}\right\|_{H^{\sigma+1}} \leq \lambda^{\sigma+1}\left\|\psi v_{\lambda}\right\|_{H^{r-1, \lambda}} .
$$

The same holds with $\left[\beta_{\mu}, \Delta_{\mathrm{g}}\right]$ replaced by $\left[\partial_{x^{\prime}},\left[\beta_{\mu}, \Delta_{\mathrm{g}}\right]\right]$, since this has the effect of differentiating the coefficients $\mathrm{g}^{i j}$ in $x^{\prime}$, which remain Lipschitz. Hence

$$
\left(\sum_{\mu}\left\|\left[\beta_{\mu}, \mathrm{g}\right] \partial_{x}^{2}\left(\psi v_{\lambda}\right)\right\|_{H^{r-2, \lambda}}^{2}\right)^{\frac{1}{2}} \lesssim \lambda\left\|\psi v_{\lambda}\right\|_{H^{r-1, \lambda}} .
$$

Next, using (14) and interpolation, we obtain for $0 \leq \sigma \leq 1$,

$$
\left(\sum_{\mu}\left\|\left(\mathrm{g}-\mathrm{g}_{\mu}\right) \partial_{x}^{2} \beta_{\mu}\left(\psi v_{\lambda}\right)\right\|_{H^{\sigma}}^{2}\right)^{\frac{1}{2}} \lesssim\left\|\partial_{x}\left(\psi v_{\lambda}\right)\right\|_{H^{\sigma}} \leq \lambda^{\sigma+1}\left\|\psi v_{\lambda}\right\|_{H^{\sigma+1, \lambda}} .
$$

Commuting with $\partial_{x^{\prime}}$ as above yields

$$
\left(\sum_{\mu}\left\|\left(\mathrm{g}-\mathrm{g}_{\mu}\right) \partial_{x}^{2} \beta_{\mu}\left(\psi v_{\lambda}\right)\right\|_{H^{r-2, \lambda}}^{2}\right)^{\frac{1}{2}} \lesssim \lambda\left\|\psi v_{\lambda}\right\|_{H^{r-1, \lambda}},
$$

completing the proof of (18).

To handle frequencies $\mu>\lambda$, we consider separately the tangential and normal components of $u_{\mu}$. Thus, we decompose

$$
\beta_{\mu}(\xi)=\Gamma_{\mu}(\xi)+\Gamma_{\mu}^{\prime}(\xi),
$$

where

$$
\operatorname{supp}\left(\Gamma_{\mu}\right) \subset\left\{\xi:\left|\xi^{\prime}\right| \leq \frac{3}{2}\left|\xi_{n}\right|\right\}, \quad \operatorname{supp}\left(\Gamma_{\mu}^{\prime}\right) \subset\left\{\xi:\left|\xi^{\prime}\right| \geq\left|\xi_{n}\right|\right\} .
$$


First consider a tangential component $u_{\mu}=\Gamma_{\mu}^{\prime}\left(\psi v_{\lambda}\right)$. The key idea is that $u_{\mu}$ and $\Delta_{\mathrm{g}_{\mu}} u_{\mu}$ are supported where $\left|\xi^{\prime}\right| \approx \mu$, whereas $\partial_{x^{\prime}}$ weighs as $\lambda$ acting on $u_{\mu}$. Consequently, for each fixed time,

$$
\left\|u_{\mu}\right\|_{L^{2}} \lesssim\left(1+\frac{\mu}{\lambda}\right)^{-N}\left\|u_{\mu}\right\|_{H^{0, \lambda}}
$$

and similarly for $\left(i \partial_{t}+\Delta_{\mathrm{g}_{\mu}}\right) u_{\mu}$. Noting that the proof of (17) works the same with with $\beta_{\mu}$ replaced by $\Gamma_{\mu}^{\prime}$, then (15), (12), and (17) together yield

$$
\left\|u_{\mu}\right\|_{L_{\mu-1}^{p} L^{q}} \lesssim\left(\frac{\mu}{\lambda}\right)^{-2} \lambda^{s}\left(\lambda^{\frac{1}{2}}\left\|v_{\lambda}\right\|_{L_{\lambda-1}^{2} L^{2}(\Omega)}+\lambda^{-\frac{1}{2}}\left\|\left(i \partial_{t}+\Delta_{\mathrm{g}}\right) v_{\lambda}\right\|_{L_{\lambda-1}^{2} L^{2}(\Omega)}\right) .
$$

We sum this over the $\mu / \lambda$ disjoint intervals of length $\mu^{-1}$ contained in $\left[0, \lambda^{-1}\right]$, and over dyadic values $\mu \geq \lambda$, to complete the proof of (10) for $\psi v_{\lambda}$ localized to frequencies $\left|\xi^{\prime}\right| \geq\left|\xi_{n}\right|$.

For a normal component $u_{\mu}=\Gamma_{\mu}\left(\psi v_{\lambda}\right)$, we do not have sufficient decay in powers of $\mu / \lambda$ to apply the above steps for large $s$. Instead, we use the fact that (15) holds for all $s \geq 0$ in this case, and deduce large $s$ results from the case $s=0$ together with Sobolev embedding. Given a triple $(p, q, s)$ satisfying $(7)$, let $q_{0} \in\left[2, \frac{n}{s}\right)$ be such that

$$
\frac{1}{q_{0}}-\frac{1}{q}=\frac{s}{n},
$$

hence $\left(p, q_{0}, 0\right)$ satisfies $(7)$. Then (15) implies

$$
\left\|u_{\mu}\right\|_{L_{\mu^{-1}}^{p} L^{q_{0}}} \lesssim \mu^{\frac{1}{2}}\left\|u_{\mu}\right\|_{L_{\mu^{-1}}^{2} L^{2}}+\left\|\left(i \partial_{t}+\Delta_{\mathrm{g}_{\mu}}\right) u_{\mu}\right\|_{L_{\mu^{-1}}^{1} L^{2}} .
$$

Summing over intervals yields, for $r \in\left[2, \frac{5}{2}\right)$, and $\mu>\lambda$,

$$
\begin{aligned}
\left\|u_{\mu}\right\|_{L_{\lambda-1}^{p} L^{q_{0}}} & \lesssim \frac{\mu}{\lambda^{\frac{1}{2}}}\left\|u_{\mu}\right\|_{L_{\lambda-1}^{2} L^{2}}+\left\|\left(i \partial_{t}+\Delta_{\mathrm{g}_{\mu}}\right) u_{\mu}\right\|_{L_{\lambda-1}^{1} L^{2}} \\
& \lesssim\left(\frac{\mu}{\lambda}\right)^{2-r}\left(\lambda^{\frac{1}{2}}\left\|u_{\mu}\right\|_{L_{\lambda-1}^{2} H^{r, \lambda}}+\left\|\left(i \partial_{t}+\Delta_{\mathrm{g}_{\mu}}\right) u_{\mu}\right\|_{L_{\lambda-1}^{1} H^{r-2, \lambda}}\right) .
\end{aligned}
$$

We apply this inequality to $\lambda^{-1} \partial_{x^{\prime}} u_{\mu}$, and observe that

$$
\left\|\left[\lambda^{-1} \partial_{x^{\prime}}, \Delta_{\mathrm{g}_{\mu}}\right] u_{\mu}\right\|_{L_{\lambda-1}^{1} H^{r-2, \lambda}} \lesssim \lambda\left\|u_{\mu}\right\|_{L_{\lambda-1}^{1} H^{r, \lambda}} \lesssim \lambda^{\frac{1}{2}}\left\|u_{\mu}\right\|_{L_{\lambda-1}^{2} H^{r, \lambda}} .
$$

This holds since multiplication by the tangential derivative $\partial_{x^{\prime}} \mathrm{g}_{\mu}$ preserves $H^{r-1, \lambda}$, provided $r<\frac{5}{2}$. Repeated application yields

$\left\|\left(\lambda^{-1} \partial_{x^{\prime}}\right)^{\alpha} u_{\mu}\right\|_{L_{\lambda-1}^{p} L^{q_{0}}} \lesssim\left(\frac{\mu}{\lambda}\right)^{2-r}\left(\lambda^{\frac{1}{2}}\left\|u_{\mu}\right\|_{L_{\lambda-1}^{2} H^{r, \lambda}}+\left\|\left(i \partial_{t}+\Delta_{\mathrm{g}_{\mu}}\right) u_{\mu}\right\|_{L_{\lambda-1}^{1} H^{r-2, \lambda}}\right)$.

We next apply Sobolev embedding to yield

$$
\begin{aligned}
\left\|u_{\mu}\right\|_{L_{\lambda-1}^{p} L^{q}} & \lesssim \mu^{\frac{s}{n}}\left\|\left|\partial_{x^{\prime}}\right|^{\frac{s(n-1)}{n}} u_{\mu}\right\|_{L_{\lambda-1}^{p} L^{q_{0}}} \\
& \lesssim \mu^{\frac{s}{n}} \lambda^{\frac{s(n-1)}{n}} \sup _{|\alpha| \leq n}\left\|\left(\lambda^{-1} \partial_{x^{\prime}}\right)^{\alpha} u_{\mu}\right\|_{L_{\lambda-1}^{p} L^{q_{0}}} \\
& \lesssim \lambda^{s}\left(\frac{\mu}{\lambda}\right)^{2+\frac{s}{n}-r}\left(\lambda^{\frac{1}{2}}\left\|u_{\mu}\right\|_{L_{\lambda-1}^{2} H^{r, \lambda}}+\left\|\left(i \partial_{t}+\Delta_{\mathrm{g}_{\mu}}\right) u_{\mu}\right\|_{L_{\lambda-1}^{1} H^{r-2, \lambda}}\right) .
\end{aligned}
$$

We choose $r \in\left(2+\frac{s}{n}, \frac{5}{2}\right)$, and apply (17). We then sum over dyadic values of $\mu>\lambda$ to establish (10) for $\psi v_{\lambda}$ localized to frequencies $\left|\xi^{\prime}\right| \leq \frac{3}{2}\left|\xi_{n}\right|$.

In proving Lemma 2.2 using the results of [20], it is convenient to work as in that paper with a first order equation. To do so, we start by rescaling the time interval of length $\mu^{-1}$ in (15) to an interval of length 1 , by considering the function 
$v(t, x)=u_{\mu}\left(\mu^{-1} t, x\right)$. This replaces $\Delta_{\mathrm{g}_{\mu}}$ by $\mu^{-1} \sum \partial_{i}\left(\mathrm{~g}_{\mu}^{i j} \partial_{j} v\right)$, which is a first order symbol for $|\xi| \approx \mu$.

We can modify this operator away from the region $|\xi| \approx \mu$ without changing the estimate (15). To fit into the framework of [20], we want to work with an operator such that solutions to the homogeneous flow remain frequency localized to $|\xi| \approx \mu$ if the initial data is supported there. For $\beta_{\mu}(\xi)$ a Littlewood-Paley cutoff to frequencies $\approx \mu$, we thus set

$$
P_{\mu}(x, D) v=\mu^{-1} \beta_{\mu}(D) \partial_{i}\left(\mathrm{~g}_{\mu}^{i j} \partial_{j} \beta_{\mu}(D) v\right)+\mu^{-1}\left(1-\beta_{\mu}(D)^{2}\right) \Delta v .
$$

Then $P_{\mu}$ is an elliptic self-adjoint operator on $\mathbb{R}^{n}$, with a symbol $p_{\mu}(x, \xi)$ such that

$$
\left\|\partial_{\xi_{i} \xi_{j}}^{2} p_{\mu}(x, \xi)-\mu^{-1} I\right\| \leq c_{0} \ll 1
$$

We will prove in the next sections the following result. Here we replace the parameter $\mu$ by $\lambda$, and $v$ by $u$, to follow the notation of [20, Theorem 2.2].

Theorem 2.3. Let $\mathrm{g}_{\lambda}^{i j}$ be obtained by truncating $\mathrm{g}^{i j}\left(x^{\prime},\left|x_{n}\right|\right)$ to frequencies $\leq c \lambda$, and define $P_{\lambda}$ as above. Suppose that $u_{\lambda}(t, x)$ is localized to spatial frequencies $|\xi| \in\left[\frac{1}{2} \lambda, \frac{5}{2} \lambda\right]$, and $p, q, s$ satisfy the conditions of Theorem 1.1. Then for small $\varepsilon$ the following holds

$$
\left\|u_{\lambda}\right\|_{L_{\varepsilon}^{p} L^{q}\left(\mathbb{R}^{n}\right)} \lesssim \lambda^{s+\frac{1}{p}}\left(\left\|u_{\lambda}\right\|_{L_{\varepsilon}^{\infty} L^{2}\left(\mathbb{R}^{n}\right)}+\left\|\left(i \partial_{t}+P_{\lambda}\right) u_{\lambda}\right\|_{L_{\varepsilon}^{1} L^{2}\left(\mathbb{R}^{n}\right)}\right) .
$$

If in addition $\hat{u}_{\lambda}$ is localized to $\left|\xi_{n}\right| \geq \frac{1}{20}|\xi|$, then (19) holds if $s \geq 0, p>2, q<\infty$, and $\frac{2}{p}+\frac{n}{q}=\frac{n}{2}-s$.

That this implies Lemma 2.2 follows from the fact that

$$
\left\|u_{\lambda}\right\|_{L_{\varepsilon}^{\infty} L^{2}\left(\mathbb{R}^{n}\right)} \lesssim\left\|u_{\lambda}\right\|_{L_{\varepsilon}^{2} L^{2}\left(\mathbb{R}^{n}\right)}+\left\|\left(i \partial_{t}+P_{\lambda}\right) u_{\lambda}\right\|_{L_{\varepsilon}^{1} L^{2}\left(\mathbb{R}^{n}\right)},
$$

which follows from self-adjointness of $P_{\lambda}$. We further note that, by the Duhamel principle, it suffices to prove the estimate for the case that $\left(i \partial_{t}+P_{\lambda}\right) u_{\lambda}=0$. In particular, it would suffice to prove (19) with $L_{\varepsilon}^{1}$ replaced by $L_{\varepsilon}^{2}$ on the right hand side, as is the case in [20, Theorem 2.2].

\section{Angular Localization}

We now proceed as in $[20, \S 3]$, and decompose $u_{\lambda}$ in the frequency domain into terms localized to angular sectors. This is done by taking a finite dyadic decomposition in the $\xi_{n}$ variable, where $\xi=\left(\xi^{\prime}, \xi_{n}\right)$. Precisely, we write $u_{\lambda}=\sum_{j=1}^{N_{\lambda}} u_{j}$, where $N_{\lambda}=\frac{1}{3} \log _{2} \lambda$, and where for $1<j<N_{\lambda}$,

$$
\operatorname{supp}\left(\widehat{u}_{j}(t, \xi)\right) \subset\left\{\left|\xi^{\prime}\right| \in\left[\frac{1}{4} \lambda, 4 \lambda\right],\left|\xi_{n}\right| \in\left[2^{-j-2} \lambda, 2^{-j+1} \lambda\right]\right\} .
$$

The "tangential" term is $u_{N_{\lambda}}$, where

$$
\operatorname{supp}\left(\widehat{u}_{N_{\lambda}}(t, \xi)\right) \subset\left\{\left|\xi^{\prime}\right| \in\left[\frac{1}{4} \lambda, 4 \lambda\right],\left|\xi_{n}\right| \leq \lambda^{\frac{2}{3}}\right\},
$$

and $u_{1}$ is localized away from tangential co-directions

$$
\operatorname{supp}\left(\widehat{u}_{1}(t, \xi)\right) \subset\left\{|\xi| \in\left[\frac{1}{2} \lambda, \frac{5}{2} \lambda\right],\left|\xi_{n}\right| \geq \frac{1}{8} \lambda\right\} .
$$

The term $u_{1}$ can be handled by the same methods as $u_{2}$, so we restrict attention to $2 \leq j \leq N_{\lambda}$. 
The energy of the solution $u_{\lambda}$ travels along bicharacteristic curves

$$
\dot{x}=d_{\xi} p_{\lambda}(x, \xi), \quad \dot{\xi}=-d_{x} p_{\lambda}(x, \xi) .
$$

For curves which satisfy $|\xi(t)| \approx \lambda$ for $t \in[0, \varepsilon]$, then $|\dot{x}| \approx 1$, and $\dot{x}_{n} \approx \lambda^{-1} \xi_{n}$. In addition, if $\left|\xi_{n}(0)\right| \approx 2^{-j} \lambda$, then we will have $\left|\xi_{n}(t)\right| \approx 2^{-j} \lambda$ for $|t| \leq \varepsilon 2^{-j}$. Setting $\theta_{j}=2^{-j}$, the function $u_{j}$ is thus localized to bicharacteristics which remain at angle $\approx \theta_{j}$ to the boundary for times up to $\varepsilon \theta_{j}$. As in [20], we will have good estimates on the term $u_{j}$ on slabs of width $\varepsilon 2^{-j}$ in $t$.

For each $j \geq 2$, we let $p_{j}(x, \xi)$ be the regularization of the symbol $p_{\lambda}(x, \xi)$, obtained by truncating the metric coefficients $\mathrm{g}_{\lambda}^{i j}(x)$ to frequencies $\leq c \lambda^{\frac{1}{2}} \theta_{j}^{-\frac{1}{2}}$.

Let $S_{j, k}$ denote the slab $\left\{(x, t): t \in\left[k \varepsilon \theta_{j},(k+1) \varepsilon \theta_{j}\right]\right\}$. The slab $S=[0, \varepsilon] \times \mathbb{R}^{n}$ is then the union of the $S_{j, k}$ as $k$ ranges over the integers from 0 to $\theta_{j}^{-1}$. Define

$$
\sigma(q)= \begin{cases}\frac{2}{3}\left(\frac{1}{2}-\frac{1}{q}\right), & n=2, \\ \frac{1}{2}-\frac{1}{q}, & n \geq 3 .\end{cases}
$$

Note that $(5)$ is equivalent to $\sigma(q) \geq \frac{1}{p}$. As in [20], the crucial matter is to now show that if $u_{j}$ satisfies the equation

$$
\left(D_{t}+P_{j}\right) u_{j}=F_{j}+G_{j},
$$

then the following estimates are valid for $2 \leq j<N_{\lambda}$

$$
\begin{aligned}
\left\|u_{j}\right\|_{L^{p} L^{q}\left(S_{j, k}\right)} \lesssim \lambda^{s+\frac{1}{p}} \theta_{j}^{\sigma(q)}( & \left\|u_{j}\right\|_{L^{\infty} L^{2}\left(S_{j, k}\right)}+\left\|F_{j}\right\|_{L^{1} L^{2}\left(S_{j, k}\right)} \\
& +\lambda^{\frac{1}{4}} \theta_{j}^{\frac{1}{4}}\left\|\left\langle\lambda^{\frac{1}{2}} \theta_{j}^{-\frac{1}{2}} x_{n}\right\rangle^{-1} u_{j}\right\|_{L^{2}\left(S_{j, k}\right)} \\
& \left.+\lambda^{-\frac{1}{4}} \theta_{j}^{-\frac{1}{4}}\left\|\left\langle\lambda^{\frac{1}{2}} \theta_{j}^{-\frac{1}{2}} x_{n}\right\rangle^{2} G_{j}\right\|_{L^{2}\left(S_{j, k}\right)}\right) .
\end{aligned}
$$

In case $j=N_{\lambda}$, that is $\theta_{j}=\lambda^{-\frac{1}{3}}$, then

$$
\left\|u_{j}\right\|_{L^{p} L^{q}\left(S_{j, k}\right)} \lesssim \lambda^{s+\frac{1}{p}} \theta_{j}^{\sigma(q)}\left(\left\|u_{j}\right\|_{L^{\infty} L^{2}\left(S_{j, k}\right)}+\left\|F_{j}+G_{j}\right\|_{L^{1} L^{2}\left(S_{j, k}\right)}\right) .
$$

Let $c_{j, k}$ denote the term in parentheses on the right of (23) (respectively the term in parentheses in (24) when $j=N_{\lambda}$ ). A modification of the arguments in [20, $\S 6]$ show that if $k(j)$ denotes any sequence of values of $k$ for which the slabs $S_{j, k(j)}$ are nested, that is, $S_{j+1, k(j+1)} \subset S_{j, k(j)}$, then

$$
\sum_{j} c_{j, k(j)}^{2} \lesssim\left\|u_{\lambda}\right\|_{L^{\infty} L^{2}(S)}^{2}+\left\|\left(i \partial_{t}+P_{\lambda}\right) u_{\lambda}\right\|_{L^{1} L^{2}(S)}^{2} .
$$

The key modification arises from the fact that the symbol $p_{\lambda}(x, \xi)$ is not homogeneous of degree 1 in $\xi$. This changes the form of the conjugation of $Q_{\mu}$ (which is the operator $P_{\lambda}$ after a space-time rescaling by $\theta_{j}$ ), by the wave packet transform $T_{\mu}$, which occurs on the bottom of $[20$, p. 137] and top of [20, p. 145]. The new relation is

$$
T_{\mu} Q_{\mu} T_{\mu}^{*}=D_{q}+i \alpha+K
$$

Here, $q$ is the symbol $p_{j}$ rescaled, and $D_{q}$ the Hamiltonian field of $q$. The real valued function $\alpha$ is defined in (31) below. The operator $D_{q}+i \alpha$ is simply the conjugation of $D_{q}$ by the unimodular function $\exp (i \psi(t, x, \xi))$. This conjugation does not affect the arguments of $[20, \S 6]$, since the only estimates used on $K$ are absolute value bounds $[20,(6.21)]$, that follow from the estimates $[20,(6.31)]$. We 
also note that the estimates in [20] use $\left(i \partial_{t}+P_{\lambda}\right) u_{\lambda} \in L^{2} L^{2}(S)$, but as noted after Theorem 2.3 above this is unimportant.

The estimates of Theorem 2.3 will then follow from (23)-(24) and the branching argument on [20, p. 118].

In the proof of (23)-(24), we will from now on work with a fixed $j$, and will abbreviate $\theta_{j}=\theta$. We work with an angle-dependent rescaled $u_{j}$, setting

$$
u(t, x)=u_{j}\left(\theta_{j} t, \theta_{j} x\right), \quad F(t, x)=\theta_{j} F_{j}\left(\theta_{j} t, \theta_{j} x\right), \quad G(t, x)=\theta_{j} G_{j}\left(\theta_{j} t, \theta_{j} x\right),
$$

and $q(x, \xi)=\theta_{j} P_{j}\left(\theta_{j} x, \theta_{j}^{-1} \xi\right)$. Set $\mu=\lambda \theta_{j}$, so that $q(x, \xi) \approx \mu$ when $|\xi| \approx \mu$. Additionally, if $|\xi| \approx \mu$, then $q(x, \xi)$ satisfies the following estimates; see [20, (4.1)].

$$
\left|\partial_{x}^{\beta} \partial_{\xi}^{\alpha} q(x, \xi)\right| \lesssim \begin{cases}\mu^{1-|\alpha|}, & \text { if }|\beta|=0 \\ c_{0}\left(1+\mu^{(|\beta|-1) / 2} \theta_{j}\left\langle\mu^{\frac{1}{2}} x_{n}\right\rangle^{-N}\right) \mu^{1-|\alpha|}, & \text { if }|\beta| \geq 1\end{cases}
$$

We then have

$$
D_{t} u-q(x, D) u=F+G,
$$

and the frequency localization condition holds

$$
\operatorname{supp}(\widehat{u}(t, \cdot)) \subset \begin{cases}\left|\xi^{\prime}\right| \in\left[\frac{1}{4} \mu, 4 \mu\right],\left|\xi_{n}\right| \in\left[\frac{1}{4} \mu \theta, 2 \mu \theta\right], & \theta>\mu^{-\frac{1}{2}} \\ \left|\xi^{\prime}\right| \in\left[\frac{1}{4} \mu, 4 \mu\right],\left|\xi_{n}\right| \leq \mu^{\frac{1}{2}}, & \theta=\mu^{-\frac{1}{2}}\end{cases}
$$

After translation in time, the estimates (23) reduce to showing that, over the slab $S=[0, \varepsilon] \times \mathbb{R}^{n}$,

$$
\begin{aligned}
\|u\|_{L^{p} L^{q}(S)} \lesssim \mu^{s+\frac{1}{p}} \theta^{\sigma(q)}( & \|u\|_{L^{\infty} L^{2}(S)}+\|F\|_{L^{1} L^{2}(S)} \\
& +\mu^{\frac{1}{4}} \theta^{\frac{1}{2}}\left\|\left\langle\mu^{\frac{1}{2}} x_{n}\right\rangle^{-1} u\right\|_{L^{2}(S)} \\
& \left.+\mu^{-\frac{1}{4}} \theta^{-\frac{1}{2}}\left\|\left\langle\mu^{\frac{1}{2}} x_{n}\right\rangle^{2} G\right\|_{L^{2}(S)}\right)
\end{aligned}
$$

The estimates in (24) reduce to showing that, for $\theta=\mu^{-\frac{1}{2}}$,

$$
\|u\|_{L^{p} L^{q}(S)} \lesssim \mu^{s+\frac{1}{p}} \theta^{\sigma(q)}\left(\|u\|_{L^{\infty} L^{2}(S)}+\|F+G\|_{L^{1} L^{2}(S)}\right) .
$$

To establish the inequalities (27) and (28), we use a wave packet transform to construct a suitable representation of $u$. Define the linear operator $T_{\mu}$ on Schwartz class functions by

$$
\left(T_{\mu} f\right)(x, \xi)=\mu^{\frac{n}{4}} \int e^{-i\langle\xi, y-x\rangle} g\left(\mu^{\frac{1}{2}}(y-x)\right) f(y) d y,
$$

where we fix $g$ a radial Schwartz class function, with $\widehat{g}$ supported in a ball of small radius $c$. Taking $\|g\|_{L^{2}\left(\mathbb{R}^{n}\right)}=(2 \pi)^{-\frac{n}{2}}$, it holds that $T_{\mu}^{*} T_{\mu}=I$ and $\left\|T_{\mu} f\right\|_{L^{2}\left(\mathbb{R}_{x, \xi}^{2 n}\right)}=$ $\|f\|_{L^{2}\left(\mathbb{R}_{y}^{n}\right)}$. We set

$$
\tilde{u}(t, x, \xi)=\left(T_{\mu} u(t, \cdot)\right)(x, \xi) .
$$

By Lemma 4.4 of [20], we may write

$$
\begin{array}{r}
\left(\partial_{t}-d_{\xi} q(x, \xi) \cdot d_{x}+d_{x} q(x, \xi) \cdot d_{\xi}+i q(x, \xi)-i \xi \cdot d_{\xi} q(x, \xi)\right) \tilde{u}(t, x, \xi) \\
=\tilde{F}(t, x, \xi)+\tilde{G}(t, x, \xi),
\end{array}
$$

where, over $\tilde{S}=[0, \varepsilon] \times \mathbb{R}_{x, \xi}^{2 n}$, the quantity

$$
\|\tilde{F}\|_{L^{1} L^{2}(\tilde{S})}+\mu^{-\frac{1}{4}} \theta^{-\frac{1}{2}}\left\|\left\langle\mu^{\frac{1}{2}} x_{n}\right\rangle^{2} \tilde{G}\right\|_{L^{2}(\tilde{S})}
$$


is bounded by the right hand side of (27) when $\theta>\mu^{-\frac{1}{2}}$, and the quantity

$$
\|\tilde{F}+\tilde{G}\|_{L^{1} L^{2}(\tilde{S})}
$$

is bounded by the right hand side of $(28)$ when $\theta=\mu^{-\frac{1}{2}}$. The proof of this lemma relies only on the bounds (26), and thus applies in our situation. Also, given the compact support of $\widehat{g}$, it can be seen that the $\xi$ support of $\tilde{u}, \tilde{F}, \tilde{G}$ is contained in a set where $\left|\xi^{\prime}\right| \approx \mu$ and $\xi_{n} \approx \theta \mu$ (or $\left|\xi_{n}\right| \lesssim \mu^{\frac{1}{2}}$ when $\theta=\mu^{-\frac{1}{2}}$ ).

Let $\Theta_{r, t}(x, \xi)$ denote the canonical transformation on $\mathbb{R}_{x, \xi}^{2 n}=T^{*}\left(\mathbb{R}_{x}^{n}\right)$ generated by the Hamiltonian flow of $q(x, \xi)$. That is, $\Theta_{r, t}(x, \xi)$ is the time $r$ solution of

$$
\dot{x}=d_{\xi} q\left(x, \xi^{\prime}\right), \quad \dot{\xi}=-d_{x} q(x, \xi),
$$

with initial conditions $(x(t), \xi(t))=(x, \xi)$. Since $q(x, \xi)$ is independent of time, $\Theta_{r, t}=\Theta_{r-t, 0}$. Also define

$$
\alpha(x, \xi)=q(x, \xi)-\xi \cdot d_{\xi} q(x, \xi), \quad \psi(t, x, \xi)=\int_{0}^{t} \alpha\left(\Theta_{s, t}(x, \xi)\right) d s .
$$

It follows by time independence of $q$ that $\int_{r}^{t} \alpha\left(\Theta_{s, t}(x, \xi)\right) d s=\psi(t-r, x, \xi)$.

Equation (29) above allows us to write

$$
\begin{aligned}
\tilde{u}(t, x, \xi)=e^{-i \psi(t, x, \xi)} & \tilde{u}\left(0, \Theta_{0, t}(x, \xi)\right) \\
& +\int_{0}^{t} e^{-i \psi(t-r, x, \xi)}\left(\tilde{F}\left(r, \Theta_{r, t}(x, \xi)\right)+\tilde{G}\left(r, \Theta_{r, t}(x, \xi)\right)\right) d r .
\end{aligned}
$$

In the next section we will establish the following estimates for solutions to the homogeneous flow equation,

Theorem 3.1. Suppose $f \in L^{2}\left(\mathbb{R}_{x, \xi}^{2 n}\right)$ is supported in a set of the form

$$
\left\{\begin{array}{l}
\left|\xi^{\prime}\right| \approx \mu,\left|\xi_{n}\right| \approx \mu \theta, \quad \theta>\mu^{-\frac{1}{2}}, \\
\left|\xi^{\prime}\right| \approx \mu,\left|\xi_{n}\right| \leq \mu^{\frac{1}{2}}, \quad \theta=\mu^{-\frac{1}{2}} .
\end{array}\right.
$$

Define $W f(t, x)=T_{\mu}^{*}\left[e^{-i \psi(t, \cdot)}\left(f \circ \Theta_{0, t}\right)\right](x)$. Then the following estimate holds for $s \geq 0, p>2$, and $q<\infty$ satisfying (5) and (7),

$$
\|W f\|_{L^{p} L^{q}(S)} \lesssim \mu^{s+\frac{1}{p}} \theta^{\sigma(q)}\|f\|_{L^{2}\left(\mathbb{R}^{2 n}\right)} .
$$

For $f \in L^{2}\left(\mathbb{R}_{x, \xi}^{2 n}\right)$ supported where $\left|\xi^{\prime}\right| \leq \mu,\left|\xi_{n}\right| \approx \mu$, estimate (33) holds with $\theta=1$, for $s \geq 0, p>2$, and $q<\infty$ satisfying (7).

Since $T_{\mu}^{*} T_{\mu}=I$, it follows by the preceeding steps and variation of parameters that this implies the estimates (28), as well as the estimates (27) in case $\tilde{G} \equiv 0$. The reduction of the estimates $(27)$ to Theorem 3.1 for $\tilde{G} \neq 0$ requires the $V_{q}^{2}$ spaces introduced by Koch and Tataru [16], and follows exactly the arguments on [20, p. 124-126]. The key fact used in that proof about the Hamiltonian flow of $q$ is that $\dot{x}_{n} \approx \theta$ on the support of $\tilde{u}(t, x, \xi)$, which holds in our case. 


\section{Homogeneous estimates}

In this section we prove Theorem 3.1. By duality, it suffices to show that

$$
\left\|W W^{*} F\right\|_{L^{p} L^{q}(S)} \lesssim \mu^{2\left(s+\frac{1}{p}\right)} \theta^{2 \sigma(q)}\|F\|_{L^{p^{\prime}} L^{q^{\prime}}(S)},
$$

where $\widehat{F}(t, \xi)$ is supported as in (32), and we recall that

$$
s=n\left(\frac{1}{2}-\frac{1}{q}\right)-\frac{2}{p}, \quad \sigma(q)= \begin{cases}\frac{2}{3}\left(\frac{1}{2}-\frac{1}{q}\right), & n=2, \\ \frac{1}{2}-\frac{1}{q}, & n \geq 3 .\end{cases}
$$

Let $W_{t}$ denote the fixed time operator $W_{t} f=\left.W f(r, x)\right|_{r=t}$. We will show that

$$
\begin{gathered}
\left\|W_{r} W_{t}^{*}\right\|_{L^{1} \rightarrow L^{\infty}} \lesssim \mu^{\frac{n}{2}}\left(\mu^{-1}+|t-r|\right)^{-\frac{n-1}{2}}\left(\mu^{-1} \theta^{-2}+|t-r|\right)^{-\frac{1}{2}}, \\
\left\|W_{r} W_{t}^{*}\right\|_{L^{2} \rightarrow L^{2}} \lesssim 1 .
\end{gathered}
$$

Interpolation of these estimates yields

$$
\left\|W_{r} W_{t}^{*}\right\|_{L^{q^{\prime}} \rightarrow L^{q}} \lesssim \mu^{\frac{n}{2}\left(1-\frac{2}{q}\right)}\left(\mu^{-1}+|t-r|\right)^{-\frac{n-1}{2}\left(1-\frac{2}{q}\right)}\left(\mu^{-1} \theta^{-2}+|t-r|\right)^{-\frac{1}{2}\left(1-\frac{2}{q}\right)} .
$$

For $n \geq 3$, we have $\frac{2}{p} \leq 1-\frac{2}{q} \leq \frac{n-1}{2}\left(1-\frac{2}{q}\right)$, hence we may ignore the term $|t-r|$ in the last factor to obtain

$$
\left\|W_{r} W_{t}^{*}\right\|_{L^{q^{\prime} \rightarrow L^{q}}} \lesssim \mu^{2\left(s+\frac{1}{p}\right)} \theta^{2 \sigma(q)}|t-r|^{-\frac{2}{p}} .
$$

In case $n=2$, we use that $\theta \leq 1$ to bound

$$
\left\|W_{r} W_{t}^{*}\right\|_{L^{q^{\prime} \rightarrow L^{q}}} \lesssim \mu^{\frac{4}{3}\left(1-\frac{2}{q}\right)} \theta^{\frac{2}{3}\left(1-\frac{2}{q}\right)}\left(\mu^{-1}+|t-r|\right)^{-\frac{2}{3}\left(1-\frac{2}{q}\right)} .
$$

Since $\frac{2}{p} \leq \frac{2}{3}\left(1-\frac{2}{q}\right)$, in this case we again have (38). In either case, the HardyLittlewood-Sobolev theorem gives (34).

In case $\theta=1$, as for the normal piece, estimate (38) follows for all $p, q$ satisfying (7) with $s \geq 0$. Hence for the normal piece the condition (5) is not necessary.

The inequality (36) follows from the fact that $T_{\mu}$ is an isometry and $\Theta_{0, t}(x, \xi)$ is a symplectomorphism, and hence preserves the measure $d x d \xi$. The remainder of this section is devoted to proving (35).

The action of $W_{r} W_{t}^{*}$ on a function $h(y)$ can be expressed as integration against an integral kernel $K(r, x ; t, y)$, defined by the formula

$$
\mu^{\frac{n}{2}} \int e^{i\langle\zeta, x-z\rangle-i \psi(r-t, x, \zeta)-i\left\langle\zeta_{t, r}, y-z_{t, r}\right\rangle} g\left(\mu^{\frac{1}{2}}\left(y-z_{t, r}\right)\right) g\left(\mu^{\frac{1}{2}}(x-z)\right) \beta_{\theta}(\zeta) d z d \zeta .
$$

Recall that $\widehat{g}$ is supported in a ball of small radius and $f(x, \xi)$ is assumed to have $\xi$ support in a set of the form (32), which is essentially preserved by the Hamiltonian flow of $q$ for time $\varepsilon$. Hence $\beta_{\theta}(\zeta)$ can be taken to be a smooth cutoff to a set of the form (32). For convenience, we take $\beta_{\theta}(\zeta)$ to be a product of a cutoff in $\zeta^{\prime}$ and a cutoff in $\zeta_{n}$.

Since $\Theta_{t, r}=\Theta_{t-r, 0}$, it suffices to consider the case $r=0$. We abbreviate $\left(z_{t, 0}, \zeta_{t, 0}\right)$ by $\left(z_{t}, \zeta_{t}\right)$, so that

$$
\partial_{t} z_{t}(z, \zeta)=d_{\zeta} q(z, \zeta), \quad \partial_{t} \zeta_{t}(z, \zeta)=-d_{z} q(z, \zeta), \quad\left(z_{0}, \zeta_{0}\right)=(z, \zeta) .
$$

The kernel $K(0, x ; t, y)$ takes the form

$$
\mu^{\frac{n}{2}} \int e^{i\langle\zeta, x-z\rangle+i \psi\left(t, z_{t}, \zeta_{t}\right)-i\left\langle\zeta_{t}, y-z_{t}\right\rangle} g\left(\mu^{\frac{1}{2}}\left(y-z_{t}\right)\right) g\left(\mu^{\frac{1}{2}}(x-z)\right) \beta_{\theta}(\zeta) d z d \zeta .
$$


Theorem 4.1. Suppose $\left(z_{t}(z, \zeta), \zeta_{t}(z, \zeta)\right)$ are defined by (39) and $d_{z}, d_{\zeta}$ denote the $z$ and $\zeta$ gradient operators. Then if $|\zeta| \approx \mu$, and $\zeta_{n} \approx \mu \theta$, or $\left|\zeta_{n}\right| \lesssim \mu^{\frac{1}{2}}$ in the case $\theta=\mu^{-\frac{1}{2}}$, the following bounds hold,

$$
\begin{array}{rr}
\left|d_{z} z_{t}-I\right| \lesssim t, & \left|d_{\zeta} z_{t}\right| \lesssim \mu^{-1} t \\
\left|d_{\zeta} \zeta_{t}-I\right| \lesssim t, & \left|d_{z} \zeta_{t}\right| \lesssim \mu
\end{array}
$$

as well as the more precise estimate

$$
\left|d_{\zeta} z_{t}-\int_{0}^{t} d_{\zeta}^{2} q\left(z_{s}, \zeta_{s}\right) d s\right| \lesssim \mu^{-1} t^{2}
$$

Furthermore, for second order derivatives we have

$$
\begin{array}{rr}
\left|d_{z}^{2} z_{t}\right| \lesssim\left\langle\mu^{\frac{1}{2}} t\right\rangle, & \left|d_{z}^{2} \zeta_{t}\right| \lesssim \mu^{\frac{3}{2}}, \\
\left|d_{z} d_{\zeta} z_{t}\right| \lesssim \mu^{-1} t\left\langle\mu^{\frac{1}{2}} t\right\rangle, & \left|d_{z} d_{\zeta} \zeta_{t}\right| \lesssim\left\langle\mu^{\frac{1}{2}} t\right\rangle .
\end{array}
$$

Finally, for $l \geq 2$ we have

$$
\mu^{l}\left|d_{\zeta}^{l} z_{t}\right|+\mu^{l-1}\left|d_{\zeta}^{l} \zeta_{t}\right| \lesssim t\left\langle\mu^{\frac{1}{2}} t\right\rangle^{l-1}
$$

Proof. The proof is a rescaled version of Theorem 5.1 and Corollary 5.2 of [20], but for completeness we sketch the details here.

Differentiating Hamilton's equations one obtains

$$
\partial_{t}\left[\begin{array}{l}
d z_{t} \\
d \zeta_{t}
\end{array}\right]=M\left(z_{t}, \zeta_{t}\right)\left[\begin{array}{l}
d z_{t} \\
d \zeta_{t}
\end{array}\right], \quad M(z, \zeta)=\left[\begin{array}{rr}
d_{z} d_{\zeta} q & d_{\zeta} d_{\zeta} q \\
-d_{z} d_{z} q & -d_{\zeta} d_{z} q
\end{array}\right]
$$

To keep all terms of the same order in $\mu$, we take the following rescaled equation,

$$
\partial_{t}\left[\begin{array}{rr}
d_{z} z_{t} & \mu d_{\zeta} z_{t} \\
\mu^{-1} d_{z} \zeta_{t} & d_{\zeta} \zeta_{t}
\end{array}\right]=M_{\mu}\left(z_{t}, \zeta_{t}\right)\left[\begin{array}{rr}
d_{z} z_{t} & \mu d_{\zeta} z_{t} \\
\mu^{-1} d_{z} \zeta_{t} & d_{\zeta} \zeta_{t}
\end{array}\right]
$$

where

$$
M_{\mu}(z, \zeta)=\left[\begin{array}{rr}
d_{z} d_{\zeta} q & \mu d_{\zeta} d_{\zeta} q \\
-\mu^{-1} d_{z} d_{z} q & -d_{\zeta} d_{z} q
\end{array}\right]
$$

The key estimate on $M_{\mu}$ is that, for $j+k=2$,

$$
\int_{0}^{t}\left|\left(d_{z}^{j} d_{\zeta}^{k} q\right)\left(z_{s}, \zeta_{s}\right)\right| d s \lesssim \begin{cases}\mu^{-1} t, & \text { if } k=2, \\ t, & \text { if } j=k=1, \\ \mu, & \text { if } j=2 .\end{cases}
$$

This follows from $(26)$ and the property $\left|\left(\partial_{t} z_{t}\right)_{n}\right| \approx \theta$ for $t \in[0, \varepsilon]$, when $\theta>\mu^{-\frac{1}{2}}$. When $\theta=\mu^{-\frac{1}{2}}$, the estimates (26) are uniform over $|\beta| \leq 2$, and (47) also follows. Gronwall's lemma now gives that

$$
\left|d_{z} z_{t}\right|+\mu\left|d_{\zeta} z_{t}\right|+\mu^{-1}\left|d_{z} \zeta_{t}\right|+\left|d_{\zeta} \zeta_{t}\right| \lesssim 1
$$

Integrating (46) and using (47) yields (41). The estimate $\left|d_{\zeta} \zeta_{t}-I\right| \lesssim t$ can then be substituted in the integral equation for $\partial_{\zeta} z_{t}$ to give (42).

To show the higher order estimates (45), we work with the equation

$$
\partial_{t}\left[\begin{array}{c}
\mu^{l} d_{\zeta}^{l} z_{t} \\
\mu^{l-1} d_{\zeta}^{l} \zeta_{t}
\end{array}\right]=M_{\mu}\left(z_{t}, \zeta_{t}\right)\left[\begin{array}{c}
\mu^{l} d_{\zeta}^{l} z_{t} \\
\mu^{l-1} d_{\zeta}^{l} \zeta_{t}
\end{array}\right]+\left[\begin{array}{c}
E_{1}(t) \\
E_{2}(t)
\end{array}\right]
$$

Here $E_{1}(t)$ is a sum of terms of the form

$$
\left(\mu^{k} d_{z}^{j} d_{\zeta}^{k+1} q\right)\left(z_{t}, \zeta_{t}\right)\left(\mu^{l_{1}} d_{\zeta}^{l_{1}} z_{t}\right) \ldots\left(\mu^{l_{j}} d_{\zeta}^{l_{j}} z_{t}\right)\left(\mu^{l_{j+1}-1} d_{\zeta}^{l_{j+1}} \zeta_{t}\right) \ldots\left(\mu^{l_{j+k}-1} d_{\zeta}^{l_{j+k}} \zeta_{t}\right) .
$$


Similarly, $E_{2}(t)$ can be written as a sum of such terms, but with the first factor replaced by $\left(\mu^{k-1} d_{z}^{j+1} d_{\zeta}^{k} q\right)\left(z_{t}, \zeta_{t}\right)$. In either case, $l_{m}<l$ for all $m$ and $l_{1}+\cdots l_{j+k}=$ $l$. The estimate (45) now follows by an inductive argument which uses the bounds

$$
\int_{0}^{t} \mu^{k-1}\left|\left(d_{z}^{j+1} d_{\zeta}^{k} q\right)\left(z_{s}, \zeta_{s}\right)\right| d s \lesssim \begin{cases}t, & \text { if } j=0 \\ \mu^{\frac{j-1}{2}}, & \text { if } j \geq 1 .\end{cases}
$$

Estimates (43) and (44) follow similarly; see the proof of Theorem 5.1 in [20].

We start the proof of (35) by noting that absolute bounds on the integrand in (40) easily yield

$$
|K(0, x ; t, y)| \lesssim \mu^{n} \theta
$$

which gives (35) for $0 \leq t \leq \mu^{-1}$. We next consider the cases $\mu^{-1} \leq t \leq \mu^{-1} \theta^{-2}$ and $\mu^{-1} \theta^{-2} \leq t \leq \varepsilon$ separately. In these two cases, we will respectively integrate by parts in (40) with the two vector fields

$$
\begin{aligned}
L^{\prime} & =\frac{1-i \mu t^{-1}\left(x-z-d_{\zeta} \zeta_{t} \cdot\left(y-z_{t}\right)\right)^{\prime} \cdot d_{\zeta^{\prime}}}{1+\mu t^{-1}\left|\left(x-z-d_{\zeta} \zeta_{t} \cdot\left(y-z_{t}\right)\right)^{\prime}\right|^{2}} \\
L & =\frac{1-i \mu t^{-1}\left(x-z-d_{\zeta} \zeta_{t} \cdot\left(y-z_{t}\right)\right) \cdot d_{\zeta}}{1+\mu t^{-1}\left|x-z-d_{\zeta} \zeta_{t} \cdot\left(y-z_{t}\right)\right|^{2}}
\end{aligned}
$$

Both $L^{\prime}$ and $L$ preserve the phase function in (40). This can be seen by noting that $\psi\left(t, z_{t}, \zeta_{t}\right)=\int_{0}^{t} \alpha\left(s, z_{s}, \zeta_{s}\right) d s$, and observing that

$$
\partial_{\zeta_{i}}\left(\int_{0}^{t} q\left(z_{s}, \zeta_{s}\right)-\zeta_{r} \cdot\left(d_{\zeta} q\right)\left(z_{s}, \zeta_{s}\right) d s\right)+\zeta_{t} \cdot \partial_{\zeta_{i}} z_{t}=0
$$

The expression vanishes at $t=0$ since $d_{\zeta} z_{0}=0$, and Hamilton's equations show that the derivative of the expression with respect to $t$ vanishes identically.

We begin with the case where $\mu^{-1} \leq t \leq \mu^{-1} \theta^{-2}$. Recall that $\beta_{\theta}(\zeta)$ is the product of smooth cutoffs to $\left|\zeta_{n}\right| \approx \theta \mu$ and $\left|\zeta^{\prime}\right| \approx \mu$. Let $\left\{\xi_{m}^{\prime}\right\}$ be a collection of $\approx(\mu t)^{\frac{n-1}{2}}$ vectors on the lattice of spacing $\mu^{\frac{1}{2}} t^{-\frac{1}{2}}$, and $\phi$ a cutoff so that

$$
\beta_{\theta}(\zeta)=\sum_{m} \beta_{\theta}(\zeta) \phi_{m}\left(\zeta^{\prime}\right),
$$

where $\phi_{m}\left(\zeta^{\prime}\right)=\phi\left(\mu^{-\frac{1}{2}} t^{\frac{1}{2}}\left(\zeta^{\prime}-\xi_{m}^{\prime}\right)\right)$.

Define $K_{m}(t, x, y)$ as the integral in (40) with $\beta_{\theta}(\zeta)$ replaced by $\beta_{\theta}(\zeta) \phi_{m}\left(\zeta^{\prime}\right)$ so that $K(0, x ; t, y)=\sum_{m} K_{m}(t, x, y)$.

By the estimates (41) and (45), we have that for $k \geq 1$,

$$
\left|\left(\mu^{\frac{1}{2}} t^{-\frac{1}{2}} d_{\zeta}\right)^{k} a\right| \lesssim 1, \quad \text { for } a(t, z, \zeta)=\mu^{\frac{1}{2}} t^{-\frac{1}{2}} z_{t} \text { or } a(t, z, \zeta)=t^{-\frac{1}{2}} d_{\zeta} \zeta_{t},
$$

which holds not just for $t \in\left[\mu^{-1}, \mu^{-1} \theta^{-2}\right]$ but for all $t \in\left[\mu^{-1}, \varepsilon\right]$. Furthermore, $\left|\left(\mu^{\frac{1}{2}} t^{-\frac{1}{2}} d_{\zeta^{\prime}}\right)^{k} \phi_{m}\left(\zeta^{\prime}\right) \beta_{\theta}(\zeta)\right| \lesssim 1$, since we do not differentiate in $\zeta_{n}$. Therefore, integration by parts yields the following upper bound on $K_{m}(t, x, y)$,

$$
\begin{aligned}
\mu^{\frac{n}{2}} \int_{\mathbb{R}^{n} \times \operatorname{supp}\left(\beta_{\theta} \phi_{m}\right)}\left(1+\mu^{\frac{1}{2}} t^{-\frac{1}{2}} \mid\right. & \left.\left(x-z-d_{\zeta} \zeta_{t} \cdot\left(y-z_{t}\right)\right)^{\prime} \mid\right)^{-N} \\
& \times\left(1+\mu^{\frac{1}{2}}\left|y-z_{t}\right|\right)^{-N}\left(1+\mu^{\frac{1}{2}}|x-z|\right)^{-N} d z d \zeta .
\end{aligned}
$$

We set $\xi_{m}=\left(\xi_{m}^{\prime}, \mu \theta\right)$. Since $t \leq \mu^{-1} \theta^{-2}$, we have for $\zeta \in \operatorname{supp}\left(\beta_{\theta} \phi_{m}\right)$,

$$
\left|\zeta-\xi_{m}\right| \lesssim \mu^{\frac{1}{2}} t^{-\frac{1}{2}}
$$


Recall that $z_{t}=z_{t}(z, \zeta)$ is the spatial component of $\Theta_{t, 0}(z, \zeta)$. We let $x_{t}^{m}=$ $z_{t}\left(x, \xi_{m}\right)$ denote the spatial component of $\Theta_{t, 0}\left(x, \xi_{m}\right)$. We then claim that, for $\zeta \in \operatorname{supp}\left(\beta_{\theta} \phi_{m}\right)$,

$$
\mu^{\frac{1}{2}} t^{-\frac{1}{2}}\left|x-z-d_{\zeta} \zeta_{t} \cdot\left(x_{t}^{m}-z_{t}\right)\right| \lesssim 1+\mu|x-z|^{2} .
$$

Assuming this for the moment, we dominate the integrand for $K_{m}$ by

$$
\left(1+\mu^{\frac{1}{2}} t^{-\frac{1}{2}}\left|\left(d_{\zeta} \zeta_{t} \cdot\left(y-x_{t}^{m}\right)\right)^{\prime}\right|\right)^{-N}\left(1+\mu^{\frac{1}{2}}\left|y-z_{t}\right|\right)^{-N}\left(1+\mu^{\frac{1}{2}}|x-z|\right)^{-N} .
$$

By (41) and (48), we have $\left|x_{t}^{m}-z_{t}\right| \lesssim \mu^{-\frac{1}{2}} t^{\frac{1}{2}}+|x-z|$. Thus, since $\left|d_{\zeta} \zeta_{t}-I\right| \lesssim|t|$, we conclude that

$$
\begin{aligned}
\mu^{\frac{1}{2}} t^{-\frac{1}{2}}\left|\left(d_{\zeta} \zeta_{t} \cdot\left(y-x_{t}^{m}\right)\right)^{\prime}\right| & \gtrsim \mu^{\frac{1}{2}} t^{-\frac{1}{2}}\left|\left(y-x_{t}^{m}\right)^{\prime}\right|-\mu^{\frac{1}{2}} t^{\frac{1}{2}}\left|y-x_{t}^{m}\right| \\
& \gtrsim \mu^{\frac{1}{2}} t^{-\frac{1}{2}}\left|\left(y-x_{t}^{m}\right)^{\prime}\right|-\mu^{\frac{1}{2}} t^{\frac{1}{2}}\left(\left|y-z_{t}\right|+|x-z|\right)-|t| .
\end{aligned}
$$

The negative terms on the right here are small compared to the last two terms in (50). Therefore, we have

$$
\left|K_{m}(t, x, y)\right| \lesssim \mu^{\frac{n+1}{2}} \theta t^{-\frac{n-1}{2}}\left(1+\mu^{\frac{1}{2}} t^{-\frac{1}{2}}\left|\left(y-x_{t}^{m}\right)^{\prime}\right|\right)^{-N},
$$

which follows by observing the rapid decay of the integrand in $z$, and that the volume of $\operatorname{supp}\left(\phi_{m} \beta_{\theta}\right)$ is comparable to $\mu^{\frac{n+1}{2}} \theta t^{-\frac{n-1}{2}}$.

We next observe that, by (42) and the estimate

$$
\left\|d_{\zeta}^{2} q(z, \zeta)-2 \mu^{-1} I\right\| \lesssim \mu^{-1}\left\|g^{i j}-\delta_{i j}\right\| \lesssim c_{0} \mu^{-1}
$$

we have that

$$
\left|\left(x_{t}^{m}-x_{t}^{l}\right)-2 \mu^{-1} t\left(\xi_{m}-\xi_{l}\right)\right| \ll \mu^{-1} t\left|\xi_{m}-\xi_{l}\right|,
$$

and since $\left|\xi_{m}-\xi_{l}\right|=\left|\xi_{m}^{\prime}-\xi_{l}^{\prime}\right|$, we conclude that

$$
\mu^{\frac{1}{2}} t^{-\frac{1}{2}}\left|\left(x_{t}^{m}-x_{t}^{l}\right)^{\prime}\right| \approx \mu^{-\frac{1}{2}} t^{\frac{1}{2}}\left|\xi_{m}^{\prime}-\xi_{l}^{\prime}\right| .
$$

Since the $\xi_{m}^{\prime}$ lie on a $\mu^{\frac{1}{2}} t^{-\frac{1}{2}}$ spaced lattice, we may sum over $m$ to obtain

$$
|K(0, x ; t, y)| \lesssim \mu^{\frac{n+1}{2}} \theta t^{-\frac{n-1}{2}} \sum_{m}\left(1+\mu^{\frac{1}{2}} t^{-\frac{1}{2}}\left|\left(y-x_{t}^{m}\right)^{\prime}\right|\right)^{-N} \lesssim \mu^{\frac{n+1}{2}} \theta t^{-\frac{n-1}{2}},
$$

yielding (35) for $\mu^{-1} \leq t \leq \mu^{-1} \theta^{-2}$.

To handle the case $\mu^{-1} \theta^{-2} \leq t \leq \varepsilon$, we modify the above proof by considering an $\mathcal{O}\left(\mu^{\frac{n}{2}} t^{\frac{n}{2}}\right)$ collection of vectors $\left\{\xi_{m}\right\}$ in a $\mu^{\frac{1}{2}} t^{-\frac{1}{2}}$ spaced lattice in $\mathbb{R}^{n}$, and an associated partition $\phi_{m}(\zeta)=\phi\left(\mu^{-\frac{1}{2}} t^{\frac{1}{2}}\left(\zeta-\xi_{m}\right)\right)$, satisfying

$$
\beta_{\theta}(\zeta)=\sum_{m} \beta_{\theta}(\zeta) \phi_{m}(\zeta)
$$

We now define $K_{m}(t, x, y)$ as the integral in (40) with $\beta_{\theta}(\zeta)$ replaced by $\beta_{\theta}(\zeta) \phi_{m}(\zeta)$. Here, since $\mu^{\frac{1}{2}} t^{-\frac{1}{2}} \leq \mu \theta$, we have

$$
\left|\left(\mu^{\frac{1}{2}} t^{-\frac{1}{2}} d_{\zeta}\right)^{k} \phi_{m}(\zeta) \beta_{\theta}(\zeta)\right| \lesssim 1 .
$$

Integrating by parts with respect to the vector field $L$ now shows that $K_{m}(t, x, y)$ is bounded by

$$
\begin{aligned}
& \mu^{\frac{n}{2}} \int_{\mathbb{R}^{n} \times \operatorname{supp}\left(\beta_{\theta} \phi_{m}\right)}\left(1+\mu^{\frac{1}{2}} t^{-\frac{1}{2}} \mid x\right.\left.-z-d_{\zeta} \zeta_{t} \cdot\left(y-z_{t}\right) \mid\right)^{-N} \\
& \times\left(1+\mu^{\frac{1}{2}}\left|y-z_{t}\right|\right)^{-N}\left(1+\mu^{\frac{1}{2}}|x-z|\right)^{-N} d z d \zeta .
\end{aligned}
$$


Using (49), which holds for any $t \in\left[\mu^{-1}, \varepsilon\right]$, we proceed as before and conclude that

$$
\left|K_{m}(t, x, y)\right| \lesssim \mu^{\frac{n}{2}} t^{-\frac{n}{2}}\left(1+\mu^{\frac{1}{2}} t^{-\frac{1}{2}}\left|y-x_{t}^{m}\right|\right)^{-N} .
$$

The $n$-dimensional analogue of (51) is valid here, so we may use the spacing of the $\xi_{m}$ as above to sum over $m$ and obtain (35) for $t>\mu^{-1} \theta^{-2}$, that is,

$$
|K(0, x ; t, y)| \lesssim \mu^{\frac{n}{2}} t^{-\frac{n}{2}} .
$$

Returning to (49), we first observe that by estimating the Taylor remainder using (43)-(45) and (48), the following holds

$$
\mu^{\frac{1}{2}} t^{-\frac{1}{2}}\left|x_{t}^{m}-z_{t}-\left(d_{z} z_{t}\right)(x-z)-\left(d_{\zeta} z_{t}\right)\left(\xi_{m}-\zeta\right)\right| \lesssim 1+\mu|x-z|^{2} .
$$

Furthermore by (41) and (48), we have

$$
\mu^{\frac{1}{2}} t^{-\frac{1}{2}}\left|\left(d_{\zeta} z_{t}\right)\left(\xi_{m}-\zeta\right)\right| \lesssim 1
$$

From the fact that $(z, \zeta) \rightarrow\left(z_{t}, \zeta_{t}\right)$ is a symplectic transformation, we have

$$
\partial_{\zeta_{i}} \zeta_{t} \cdot \partial_{z_{j}} z_{t}-\partial_{\zeta_{i}} z_{t} \cdot \partial_{z_{j}} \zeta_{t}=\delta_{i j}
$$

where - pairs the $z_{t}$ and $\zeta_{t}$ indices. Lastly, by (41),

$$
\mu^{\frac{1}{2}} t^{-\frac{1}{2}}\left|d_{\zeta} z_{t}\right|\left|d_{z} \zeta_{t}\right||x-z| \lesssim \mu^{\frac{1}{2}} t^{\frac{1}{2}}|x-z| \leq \mu^{\frac{1}{2}}|x-z| .
$$

These facts now combine to yield (49).

\section{Applications to Semilinear SchröDinger equations on EXterior DOMAINS}

In this section, we assume that $\Omega=\mathbb{R}^{3} \backslash \mathcal{K}$ is the domain exterior to a smooth non-trapping obstacle $\mathcal{K}$ (or any exterior domain where (4) holds). We consider the initial value problem for the following family of semilinear Schrödinger equations in $3+1$ dimensions,

$$
i \partial_{t} u+\Delta u \pm|u|^{r-1} u=0, \quad u(0, x)=f(x),
$$

satisfying homogeneous Dirichlet or Neumann boundary conditions

$$
\left.u(t, x)\right|_{\partial \Omega}=0, \quad \text { or }\left.\quad \partial_{\nu} u(t, x)\right|_{\partial \Omega}=0 .
$$

Precisely, by a solution to (52)-(53), we understand that, with $F(u)= \pm|u|^{r-1} u$, and $u(t)$ denoting the function $u(t, \cdot)$,

$$
u(t)=e^{i t \Delta} f+i \int_{0}^{t} e^{i(t-s) \Delta} F(u(s)) d s,
$$

where $\exp (i t \Delta)$ is the unitary Schrödinger propagator defined using the Dirichlet or Neumann spectral resolution. Defocusing means that $F(u)=-|u|^{r-1} u$. Since we will work with $H^{1}$ data, the boundary conditions required of the initial data in the Dirichlet case are that $f$ vanish on $\partial \Omega$; in the Neumann case the boundary conditions are void, i.e. $f$ is the restriction to $\Omega$ of a general function in $H^{1}\left(\mathbb{R}^{3}\right)$.

Planchon and Vega showed in [17] that, for $1<r<5$ and defocusing nonlinearities, one has global existence of solutions to the Dirichlet problem for $f \in H^{1}$. A crucial ingredient in their proof was the estimate in Theorem 1.1 with $p=q=4$ and $s=\frac{1}{4}$. They combined this with local smoothing estimates near the boundary 
to obtain well-posedness of solutions for time $T>0$ depending on $\|f\|_{H^{1}}$. Conservation of energy and mass

$$
\begin{aligned}
\int_{\Omega} \frac{1}{2}\left|d_{x} u(t)\right|^{2}+\frac{1}{r+1}|u(t)|^{r+1} d x & =\text { constant in } t \\
\int_{\Omega}|u(t)|^{2} d x & =\text { constant in } t
\end{aligned}
$$

can then be used to establish global existence of solutions. Given that Theorem 1.1 holds for either Dirichlet or Neumann boundary conditions, we remark that our estimates can be used in the same way to obtain global existence of the solution to the Neumann problem.

In the critical case $r=5$, local well-posedness for solutions with $f \in H^{1}$, and global well-posedness for small data, was proved by Ivanovici in [12], under the assumption that $\mathcal{K}$ is strictly convex, and $u$ satisfies Dirichlet conditions. These assumptions are necessary for the Melrose-Taylor parametrix construction used to establish Strichartz estimates in [12]. The Strichartz estimates were in fact shown to hold in this setting for the full range of $p, q$ satisfying (7), provided $p>2$. Recently, Ivanovici and Planchon in [13] extended the well-posedness results to the case of general non-trapping $\mathcal{K}$, for both Dirichlet and Neumann conditions, using certain $L_{x}^{5} L_{t}^{2}$ estimates from [20].

Here, we use our estimates to give a simple proof of the well-posedness results for $H^{1}$ data for the critical case $r=5$, and general non-trapping $\mathcal{K}$. The proof proceeds by a contraction argument using a $L_{t}^{4} L_{x}^{\infty}$ Strichartz estimate. As in [13], the local results are valid for the Dirichlet and Neumann cases, and the proof yields scattering for small data in the Dirichlet case. Precisely, we establish the following.

Theorem 5.1. Suppose $f \in H^{1}(\Omega)$, where $\left.f\right|_{\partial \Omega}=0$ if Dirichlet conditions are assumed. Then there exists $T>0$, and a solution $u(t, x)$ to equation (54) with $r=5$ on $[-T, T] \times \Omega$, satisfying

$$
u \in X \equiv C\left([-T, T] ; H^{1}(\Omega)\right) \cap L^{4}\left([-T, T] ; L^{\infty}(\Omega)\right),
$$

and the solution is uniquely determined in this function space. Furthermore, if the data satisfies $\|f\|_{H^{1}} \leq \varepsilon$ for $\varepsilon$ sufficiently small, one can take $T=\infty$ in the case of Dirichlet conditions, and $T=1$ for Neumann conditions.

The key ingredient in Theorem 5.1 will be the following Strichartz estimate for $u$ given by formula (54), and with $f$ and $F$ satisfying the given boundary condition,

$$
\|u\|_{L_{T}^{4} L^{\infty}} \lesssim\|f\|_{H^{1}}+\|F\|_{L_{T}^{1} H^{1}} .
$$

Given (55), one applies differentiation and Hölder's inequality to obtain

$$
\left\||u|^{4} u\right\|_{L_{T}^{1} H^{1}} \lesssim\|u\|_{L_{T}^{4} L^{\infty}}^{4}\|u\|_{L_{T}^{\infty} H^{1}},
$$

noting that $|u|^{4} u$ satisfies Dirichlet conditions if $u$ does.

We then pose $u=u_{0}+v$, where $u_{0}(t)=\exp (i t \Delta) f$, and take $T$ small enough so that $\left\|u_{0}\right\|_{L_{T}^{4} L^{\infty}}<c$, for $c$ to be determined. Estimates (55) and (56), together with conservation of the $H^{1}$ norm under $\exp (i t \Delta)$, show that, for small $c$, the map

$$
v \rightarrow \int_{0}^{t} e^{i(t-s) \Delta}\left(\left|u_{0}(s)+v(s)\right|^{4}\left(u_{0}(s)+v(s)\right)\right) d s
$$


maps the ball $\|v\|_{X} \leq c$ into itself. Similar analysis shows that the map is in fact a contraction on this ball, for small $c$, yielding a fixed point $v$. If $\|f\|_{H^{1}} \leq \varepsilon$, then one can take $T=\infty$ for the Dirichlet case, or $T=1$ for the Neumann case.

For defocusing Neumann, energy and mass conservation then yield global existence. For small norm Dirichlet data, the proof implies $|u|^{4} u \in L^{1}\left(\mathbb{R}, H^{1}(\Omega)\right)$. This yields that such solutions scatter, in the sense that they asymptotically approach in the $H^{1}$ norm a solution to the homogeneous equation.

In establishing (55), it suffices by the Duhamel principle to consider $F=0$. The proof of (55) will be obtained from the following cases of Theorem 1.1,

$$
\|u\|_{L^{12} L^{9}} \lesssim\|f\|_{H^{1}}, \quad \quad\|u\|_{L^{3} L^{9}} \lesssim\|f\|_{H^{\frac{1}{2}}} .
$$

The second estimate could be expressed as controlling the $L^{3} W^{\frac{1}{2}, 9}$ norm of $u$ in terms of $\|f\|_{H^{1}}$, and we would then apply a fractional Gagliardo-Nirenberg inequality to control $\|u(t)\|_{L^{\infty}}$ by interpolating $L^{9}$ and $W^{\frac{1}{2}, 9}$. We can avoid dealing with fractional $L^{p}$ Sobolev spaces on exterior domains, however, by carrying out the same steps more directly. The interpolation we will use is the following.

Lemma 5.2. Suppose that $\alpha_{1}, \alpha_{2}>0$, and $u=\sum_{j=0}^{\infty} u_{j}$, where

$$
\left\|u_{j}\right\|_{L^{\infty}} \leq \min \left(2^{-j \alpha_{1}} \rho_{1}, 2^{j \alpha_{2}} \rho_{2}\right) \text {. }
$$

Then

$$
\|u\|_{L^{\infty}} \leq C_{\alpha_{1}, \alpha_{2}} \rho_{1}^{\frac{\alpha_{2}}{\alpha_{1}+\alpha_{2}}} \rho_{2}^{\frac{\alpha_{1}}{\alpha_{1}+\alpha_{2}}} .
$$

Proof. The proof follows by summing the smaller of the bounds, i.e. separating the sum depending on whether $2^{j} \geq\left(\rho_{1} / \rho_{2}\right)^{\frac{1}{\alpha_{1}+\alpha_{2}}}$ or not. The bound applies with

$$
C_{\alpha_{1}, \alpha_{2}}=\frac{2^{\alpha_{1}}}{2^{\alpha_{1}}-1}+\frac{2^{\alpha_{2}}}{2^{\alpha_{2}}-1} .
$$

We next take a Littlewood-Paley decomposition of the initial data

$$
f=\sum_{j=1}^{\infty} \beta\left(2^{-2 j} H\right) f+\beta_{0}(H) f,
$$

where $\beta(s)$ is supported in the interval $s \in\left[\frac{1}{2}, \frac{9}{2}\right]$, and $1=\beta_{0}(s)+\sum_{j=0}^{\infty} \beta\left(2^{-2 j} s\right)$ for $s \geq 0$. Here, $H$ denotes $-\Delta$ with either Dirichlet or Neumann conditions. Set

$$
f_{j}=e^{2^{-2 j} H} \beta\left(2^{-2 j} H\right) f, \quad f_{0}=e^{H} \beta_{0}(H) f .
$$

By the spectral localization,

$$
\sum_{j=0}^{\infty}\left\|f_{j}\right\|_{H^{1}}^{2} \lesssim\|f\|_{H^{1}}^{2}
$$

and we may write $u(t)=\sum_{j=0}^{\infty} u_{j}(t)$, where

$$
u_{j}(t)=e^{-2^{-2 j} H} e^{-i t H} f_{j}, \quad u_{0}(t)=e^{-H} e^{-i t H} f_{0} .
$$

By the ultracontractivity estimate for $H$ on exterior domains (see Theorem 2.4.2 and the ensuing comments in [7], where $\mu=3$ in our case), we can bound

$$
\left\|u_{j}(t)\right\|_{L^{\infty}} \lesssim 2^{\frac{j}{3}}\left\|e^{-i t H} f_{j}\right\|_{L^{9}} .
$$


Together with the case $(p, q, s)=\left(3,9, \frac{1}{2}\right)$ of Theorem 1.1, we have

$$
\left\|2^{-\frac{j}{3}} u_{j}\right\|_{L^{3} L^{\infty}} \lesssim\left\|e^{-i t H} f_{j}\right\|_{L^{3} L^{9}} \lesssim\left\|f_{j}\right\|_{H^{\frac{1}{2}}} \leq 2^{-\frac{j}{2}}\left\|f_{j}\right\|_{H^{1}}
$$

which we combine with Minkowski's inequality to yield

$$
\left(\int\left(\sum_{j=0}^{\infty}\left\|2^{\frac{j}{6}} u_{j}(t)\right\|_{L^{\infty}}^{2}\right)^{\frac{3}{2}} d t\right)^{\frac{1}{3}} \leq\left(\sum_{j=0}^{\infty}\left\|2^{\frac{j}{6}} u_{j}\right\|_{L^{3} L^{\infty}}^{2}\right)^{\frac{1}{2}} \lesssim\|f\|_{H^{1}}^{2} .
$$

In particular,

$$
\sup _{j} 2^{\frac{j}{6}}\left\|u_{j}(t)\right\|_{L^{\infty}} \leq \rho_{1}(t), \quad\left\|\rho_{1}\right\|_{L^{3}} \lesssim\|f\|_{H^{1}} .
$$

Similar considerations, using the case $(p, q, s)=(12,9,1)$ of Theorem 1.1, yield

$$
\sup _{j} 2^{-\frac{j}{3}}\left\|u_{j}(t)\right\|_{L^{\infty}} \leq \rho_{2}(t), \quad\left\|\rho_{2}\right\|_{L^{12}} \lesssim\|f\|_{H^{1}} .
$$

Lemma 5.2 now applies to give the bound

$$
\|u(t)\|_{L^{\infty}} \lesssim \rho_{1}(t)^{\frac{2}{3}} \rho_{2}(t)^{\frac{1}{3}} .
$$

Applying Hölder's inequality with the dual indices $\left(\frac{9}{8}, 9\right)$ now yields

$$
\|u\|_{L^{4} L^{\infty}}^{4} \lesssim \int \rho_{1}(t)^{\frac{8}{3}} \rho_{2}(t)^{\frac{4}{3}} d t \lesssim\left\|\rho_{1}\right\|_{L^{3}}^{\frac{8}{3}}\left\|\rho_{2}\right\|_{L^{12}}^{\frac{4}{3}} \lesssim\|f\|_{H^{1}}^{4} .
$$

\section{Applications to semilinear Schrödinger equations on compact MANIFOLDS}

In this section we consider a compact 3-dimensional Riemannian manifold $\Omega$ with boundary. We assume $G:[0, \infty) \rightarrow \mathbb{R}$ is bounded below, with $G(0)=0$, and that

$$
\left|G^{\prime}(r)\right|+r\left|G^{\prime \prime}(r)\right| \lesssim\langle r\rangle^{\frac{1}{5}}
$$

We set $F(u)=G^{\prime}\left(|u|^{2}\right) u$, so that

$$
|F(u)| \leq\langle u\rangle^{2 / 5}|u|, \quad\left|d_{u} F(u)\right| \leq\langle u\rangle^{2 / 5} .
$$

We prove existence, uniqueness, and energy conservation, for initial data $u\left(t_{0}\right) \in$ $H^{1}(\Omega)$, to the semilinear Schrödinger equation

$$
i \partial_{t} u+\Delta u=F(u),\left.\quad u\right|_{t=t_{0}}=u\left(t_{0}\right)
$$

satisfying homogeneous Dirichlet or Neumann boundary conditions (53). As above, by a solution to (58) we understand that its integral form holds,

$$
u(t)=e^{i\left(t-t_{0}\right) \Delta}\left(u\left(t_{0}\right)-i \int_{t_{0}}^{t} e^{-i\left(s-t_{0}\right) \Delta} F(u(s)) d s\right) .
$$

This formulation is seen to be independent of $t_{0}$; that is, if $u$ solves (59) on an interval for some $t_{0}$ then it solves the same equation for all $t_{0}$ in that interval.

The key estimates we use involve values of $(p, q)$ which do not satisfy (5). In this case, the method of proof yields estimates with a loss of derivatives relative to the scale invariant value of $s$ from (7). In particular, the following analogue of Theorem 2.1 loses $\frac{1}{q}$ derivatives relative to the case of manifolds without boundary considered in [5]. Additionally, there are logarithmic losses due to the endpoint $p=2$ and $q=\infty$. 
Lemma 6.1. Let $n=3$, and suppose that for all $t, u_{\lambda}(t, \cdot)$ is spectrally localized for $-\Delta_{\mathrm{g}}$ to the range $\left[\frac{1}{4} \lambda^{2}, 4 \lambda^{2}\right]$. Then the following estimate holds, uniformly for $6 \leq q \leq \infty$, where $F_{\lambda}=\left(i \partial_{t}+\Delta_{\mathrm{g}}\right) u_{\lambda}$,

$$
\left\|u_{\lambda}\right\|_{L_{\lambda-1}^{2} L^{q}(\Omega)} \leq C \lambda^{\frac{1}{2}-\frac{2}{q}}(\log \lambda)^{2}\left(\lambda^{\frac{1}{2}}\left\|u_{\lambda}\right\|_{L_{\lambda-1}^{2} L^{2}(\Omega)}+\lambda^{-\frac{1}{2}}\left\|F_{\lambda}\right\|_{L_{\lambda-1}^{2} L^{2}(\Omega)}\right) .
$$

Proof. We start by noting that the reduction of Theorem 2.1 to Theorem 2.3 holds with uniform constant over $q \geq 6$ with $p=2$. In particular, in the handling of the normal piece, $q_{0}=6$ for $p=2$, and $s \leq \frac{1}{2}$ in our estimates, so the use of Sobolev embedding works for that piece. Thus, (60) is a consequence of the estimate

$$
\left\|u_{\lambda}\right\|_{L_{\varepsilon}^{2} L^{q}} \leq C \lambda^{1-\frac{2}{q}}(\log \lambda)^{2}\left(\left\|u_{\lambda}\right\|_{L_{\varepsilon}^{\infty} L^{2}}+\left\|\left(i \partial_{t}+P_{\lambda}\right) u_{\lambda}\right\|_{L_{\varepsilon}^{1} L^{2}}\right),
$$

together with the following estimate, valid if $\hat{u}_{\lambda}$ is localized to $\left|\xi_{n}\right| \geq \frac{1}{20}|\xi|$,

$$
\left\|u_{\lambda}\right\|_{L_{\varepsilon}^{2} L^{q}} \leq C \lambda^{1-\frac{3}{q}}(\log \lambda)^{2}\left(\left\|u_{\lambda}\right\|_{L_{\varepsilon}^{\infty} L^{2}}+\left\|\left(i \partial_{t}+P_{\lambda}\right) u_{\lambda}\right\|_{L_{\varepsilon}^{1} L^{2}}\right)
$$

where $P_{\lambda}$ is as in Theorem 2.3. These estimates in turn follows as a consequence of the following analogue of (23)

$$
\begin{aligned}
\left\|u_{j}\right\|_{L^{2} L^{q}\left(S_{j, k}\right)} \leq C \lambda^{1-\frac{3}{q}}(\log \lambda)^{\frac{3}{2}} \theta_{j}^{\frac{1}{2}-\frac{3}{q}} & \left\|u_{j}\right\|_{L^{\infty} L^{2}\left(S_{j, k}\right)}+\left\|F_{j}\right\|_{L^{1} L^{2}\left(S_{j, k}\right)} \\
& +\lambda^{\frac{1}{4}} \theta_{j}^{\frac{1}{4}}\left\|\left\langle\lambda^{\frac{1}{2}} \theta_{j}^{-\frac{1}{2}} x_{n}\right\rangle^{-1} u_{j}\right\|_{L^{2}\left(S_{j, k}\right)} \\
& \left.+\lambda^{-\frac{1}{4}} \theta_{j}^{-\frac{1}{4}}\left\|\left\langle\lambda^{\frac{1}{2}} \theta_{j}^{-\frac{1}{2}} x_{n}\right\rangle^{2} G_{j}\right\|_{L^{2}\left(S_{j, k}\right)}\right) .
\end{aligned}
$$

To see this, we note that for $p=2$, the branching argument $[20$, p.118] requires $\theta_{j}^{\frac{1}{2}}$ to converge, and the remaining term $\theta_{j}^{-\frac{3}{q}}$ is bounded by $\lambda^{\frac{1}{q}}$. The additional $\operatorname{loss}$ of $(\log \lambda)^{\frac{1}{2}}$ here comes from the fact that there are $\sim \log \lambda$ terms $j$ in the decomposition of $u_{\lambda}=\sum_{j} u_{j}$. We thus have, uniformly in $q$,

$$
\left\|u_{\lambda}\right\|_{L_{\varepsilon}^{2} L^{q}} \lesssim(\log \lambda)^{\frac{1}{2}}\left\|\left(\sum_{j}\left|u_{j}\right|^{2}\right)^{\frac{1}{2}}\right\|_{L_{\varepsilon}^{2} L^{q}}
$$

and it is the norm on the right hand side that is controlled by the branching argument.

The estimate (61) is scale invariant; scaling by $\theta$ reduces it to the following analogue of (27), for angularly localized $u$ satisfying $\left(D_{t}-q(x, D)\right) u=F+G$,

$$
\begin{aligned}
\|u\|_{L^{2} L^{q}(S)} \lesssim \mu^{1-\frac{3}{q}}(\log \mu)^{\frac{3}{2}} \theta^{\frac{1}{2}-\frac{3}{q}} & \|u\|_{L^{\infty} L^{2}(S)}+\|F\|_{L^{1} L^{2}(S)} \\
& +\mu^{\frac{1}{4}} \theta^{\frac{1}{2}}\left\|\left\langle\mu^{\frac{1}{2}} x_{n}\right\rangle^{-1} u\right\|_{L^{2}(S)} \\
& \left.+\mu^{-\frac{1}{4}} \theta^{-\frac{1}{2}}\left\|\left\langle\mu^{\frac{1}{2}} x_{n}\right\rangle^{2} G\right\|_{L^{2}(S)}\right),
\end{aligned}
$$

where we used that $\log \mu \approx \log \lambda$.

The reduction of (62) to homogeneous estimates, that is, bounds on the operator $W$ of (33), involves a $\operatorname{loss}$ of $\log \mu$ due to the fact that $p=2$. This comes from the use of the $V_{q}^{2}$ spaces introduced by Koch and Tataru [16], where the subscript $q$ refers to the Hamiltonian flow for $q(x, \xi)$. In case $p=2$, one needs to control the 2-atomic norm $U_{q}^{2}$ of $\tilde{u}$, whereas $V_{q}^{2} \subset U_{q}^{p}$ only for $p>2$. To proceed, we note that in the atomic decomposition argument of [16, Lemma 6.4], we may truncate the sum $u=\sum_{n} v_{n}$ to $n \lesssim \log \mu$, since the error is bounded in $L^{\infty} L^{2}$ by $\mu^{-N}$, and its contribution thus may be estimated in the desired norm using Sobolev embedding. 
Each term $v_{n}$ is uniformly bounded in $U_{q}^{2}$, hence the $U_{q}^{2}$ norm of the truncated sum is $\lesssim \log \mu$.

We are thus reduced to establishing the following analogue of (33),

$$
\|W f\|_{L^{2} L^{q}(S)} \leq C \mu^{1-\frac{3}{q}}(\log \mu)^{\frac{1}{2}} \theta^{\frac{1}{2}-\frac{3}{q}}\|f\|_{L^{2}\left(\mathbb{R}^{2 n}\right)} .
$$

To establish (63), we consider $W W^{*}$ as in the proof of Theorem 3.1. Taking $n=3$ in (37), we note the following integral bound for $6 \leq q \leq \infty, \mu$ and $\theta$ as above,

$$
\int_{0}^{1}\left(\mu^{-1}+t\right)^{-\left(1-\frac{2}{q}\right)}\left(\mu^{-1} \theta^{-2}+t\right)^{-\frac{1}{2}\left(1-\frac{2}{q}\right)} d t \leq C \mu^{\frac{3}{2}\left(1-\frac{2}{q}\right)-1}(\log \mu) \theta^{1-\frac{6}{q}},
$$

where $C$ is uniformly bounded. The estimate (63) follows by Schur's lemma.

We use Lemma 6.1 to deduce the following analogue of Lemma 3.6 of [5]. This version is weaker, both in the logarithmic loss and the loss of $\lambda^{\frac{1}{q}}$, but is sufficient for our purposes. From now on, we let $u_{\lambda}=\beta\left(\lambda^{-2} H\right) u$ denote a Littlewood-Paley decomposition of $u$, where $\lambda=2^{k}$ and $k \geq 1$. The term $k=0$ contains the low frequency terms of $u$, and the bounds for this term will follow similarly to $k=1$.

Lemma 6.2. Let $u$ solve (59). Then there are $C<\infty$ and $\varepsilon>0$ such that, uniformly for $6 \leq q \leq \infty$, the following holds on any time interval $[0, T]$ with $\lambda^{-1} \leq T \leq 1$,

$$
\left\|u_{\lambda}\right\|_{L^{2}\left([0, T], L^{q}\right)} \leq C \lambda^{-\frac{2}{q}}(\log \lambda)^{2}\left(\left\|u_{\lambda}\right\|_{L^{2}\left([0, T], H^{1}\right)}+\lambda^{-\varepsilon}\left\langle\|u\|_{L^{\infty}\left([0, T], H^{1}\right)}\right\rangle^{7 / 5}\right) .
$$

Proof. We divide $[0, T]$ into subintervals of length $\lambda^{-1}$. We apply (60) on each such subinterval, and square sum over subintervals to obtain

$$
\left\|u_{\lambda}\right\|_{L^{2}\left([0, T], L^{q}\right)} \leq C \lambda^{-\frac{2}{q}}(\log \lambda)^{2}\left(\left\|u_{\lambda}\right\|_{L^{2}\left([0, T], H^{1}\right)}+\left\|F_{\lambda}\right\|_{L^{2}\left([0, T], L^{2}\right)}\right),
$$

where $F_{\lambda}=F(u)_{\lambda}$. We now take

$$
\alpha=\frac{2}{5}, \quad r=\frac{6}{3+\alpha}, \quad \varepsilon=3\left(\frac{1}{r}-\frac{1}{2}\right)-1,
$$

and observe that

$$
\begin{aligned}
\left\|F_{\lambda}\right\|_{L^{\infty} L^{2}} & \lesssim \lambda^{-\varepsilon}\|F\|_{L^{\infty} W^{1, r}} \\
& \lesssim \lambda^{-\varepsilon}\left\|\langle u\rangle^{\alpha}\left(\left|d_{x} u\right|+\langle u\rangle\right)\right\|_{L^{\infty} L^{r}} \\
& \lesssim \lambda^{-\varepsilon}\|\langle u\rangle\|_{L^{\infty} L^{6}}^{\alpha}\left\|\left(\left|d_{x} u\right|+\langle u\rangle\right)\right\|_{L^{\infty} L^{2}} \\
& \lesssim \lambda^{-\varepsilon}\left(1+\|u\|_{L^{\infty} H^{1}}\right)^{\alpha+1}
\end{aligned}
$$

Sobolev embedding yields $\left\|u_{<T^{-1}}\right\|_{L_{T}^{2} L^{q}} \lesssim T^{-\frac{1}{2}}\|u\|_{L_{T}^{2} H^{1}} \lesssim\|u\|_{L_{T}^{\infty} H^{1}}$, where $u_{<T^{-1}}$ denotes the sum of $u_{\lambda}$ over $\lambda<T^{-1}$. Summing (64) over $\lambda=2^{-k}$, and using Cauchy-Schwarz over $k$, we conclude that, with $C$ uniform over $q \geq 6$,

$$
\|u\|_{L^{2}\left([0, T], L^{q}\right)} \leq C q^{\frac{5}{2}}\left(1+\|u\|_{L^{\infty} H^{1}}\right)^{7 / 5}
$$


Suppose now that $u$ satisfies (59) on a time interval $[0, T]$, where $u\left(t_{0}\right) \in H^{1}(\Omega)$. For sufficiently regular solutions $u$, we have the conservation laws

$$
\begin{aligned}
\int_{\Omega}|u(t)|^{2} & =\int_{\Omega}\left|u\left(t_{0}\right)\right|^{2} \\
\int_{\Omega}\left|d_{x} u(t)\right|_{\mathrm{g}}^{2}+G\left(|u(t)|^{2}\right) & =\int_{\Omega}\left|d_{x} u\left(t_{0}\right)\right|_{\mathrm{g}}^{2}+G\left(\left|u\left(t_{0}\right)\right|^{2}\right)
\end{aligned}
$$

In particular, since $-C \leq G(r) \leq C\langle r\rangle^{\frac{6}{5}}$, it follows that $\|u\|_{L^{\infty}\left([0, T], H^{1}\right)} \lesssim 1+$ $\left\|u\left(t_{0}\right)\right\|_{H^{1}}$, uniformly in $T$.

In the following proof, we assume a priori that $u \in L^{\infty} H^{1}$ and prove uniqueness of such solutions. The existence of bounded energy solutions, and energy conservation, is then proved by a weak-limit argument.

Theorem 6.3. For each data $f \in H^{1}(\Omega)$, and all $T>0$, there exists a unique solution $u$ to the equation (59), subject to the condition $u \in L^{\infty}\left([0, T], H^{1}(\Omega)\right)$. Furthermore, the solution satisfies the conservation laws (66).

Proof. We start with the uniqueness of solutions. Since $u \in L^{\infty}\left([0, T], H^{1}\right)$ it follows by Sobolev embedding that $F(u) \in L^{\infty} L^{2}$, so $u \in C\left([0, T], L^{2}\right)$, and by interpolation $u \in C\left([0, T], H^{s}\right)$ for all $s<1$. Repeating this argument shows that the term in parentheses in (59) belongs to $C^{1}\left([0, T], L^{2}\right)$.

Let $u$ and $v$ be two solutions to $(59)$, with $u(0)=v(0)$. By unitarity of $\exp ($ it $\Delta)$,

$$
\begin{aligned}
\frac{d}{d t}\|u(t)-v(t)\|_{L^{2}}^{2} & =\frac{d}{d t}\left\|e^{-i t \Delta}(u(t)-v(t))\right\|_{L^{2}}^{2} \\
& =2 \operatorname{Im}\langle F(u(t))-F(v(t)), u(t)-v(t)\rangle \\
& \leq C \int_{\Omega}\left(\langle u(t)\rangle^{2 / 5}+\langle v(t)\rangle^{2 / 5}\right)|u(t)-v(t)|^{2} \\
& \leq C\left(1+\|u(t)\|_{L^{2 q / 5}}+\|v(t)\|_{L^{2 q / 5}}\right)^{\frac{2}{5}}\|u(t)-v(t)\|_{L^{2 q^{\prime}}}^{2}
\end{aligned}
$$

provided $q \geq 5 / 2$. Since $\|u(t)-v(t)\|_{L^{6}} \leq C$, we may interpolate to bound

$$
\|u(t)-v(t)\|_{L^{2 q^{\prime}}} \leq C\|u(t)-v(t)\|_{L^{2}}^{1-\frac{3}{2 q}} .
$$

Setting $g(t)=\|u(t)-v(t)\|_{L^{2}}^{2}$, and noting $g(0)=0$, we have upon integrating that

$$
g(\tau)^{\frac{3}{2 q}} \leq \frac{C}{q} \int_{0}^{\tau}\left(\|u(t)\|_{L^{2 q / 5}}^{\frac{2}{5}}+\|v(t)\|_{L^{2 q / 5}}^{\frac{2}{5}}\right) d t+\frac{C \tau}{q}
$$

By Hölder's inequality and (65), for $\tau \in[0, T]$

$$
\int_{0}^{\tau}\|u(t)\|_{L^{2 q / 5}}^{\frac{2}{5}} d t \leq \tau^{\frac{4}{5}}\|u\|_{L^{2}\left([0, T], L^{2 q / 5}\right)}^{\frac{2}{5}} \leq C \tau^{\frac{4}{5}} q .
$$

Consequently,

$$
g(\tau) \leq\left(C \tau^{\frac{4}{5}}+\frac{C \tau}{q}\right)^{\frac{2 q}{3}}
$$

which goes to 0 as $q \rightarrow \infty$, provided $\tau$ is small depending on $C$. Repeating the argument yields uniqueness on $[0, T]$.

To establish existence and energy conservation for (59) with $H^{1}$ data, we let $G_{j}(r)$ be a family of smooth, compactly supported real valued functions on $[0, \infty)$, 
uniformly bounded below, such that $G_{j}(r)$ and $G_{j}^{\prime}(r)$ converge uniformly on compact sets to $G(r)$ and $G^{\prime}(r)$. Additionally, we require that (57) holds uniformly over $j$ for $G=G_{j}$.

We fix a time $t_{0}$ and initial data $u\left(t_{0}\right) \in H^{1}(\Omega)$, and let $u_{j}(t)$ solve (59) where $F$ is replaced by $F_{j}=G_{j}^{\prime}\left(|u|^{2}\right) u$. We assume for the moment that $u_{j}$ exists globally in time, and satisfies the conservation law (66), with $G$ replaced by $G_{j}$. In particular

$$
\left\|u_{j}\right\|_{L^{\infty} H^{1}} \leq C\left(1+\left\|u\left(t_{0}\right)\right\|_{H^{1}}\right) \quad \text { uniformly over } j .
$$

By (59), $\exp \left(-i\left(t-t_{0}\right) \Delta\right) u_{j}(t)$ is uniformly bounded in $C^{1} L^{2} \cap L^{\infty} H^{1} \subset C^{\frac{1}{2}} H^{\frac{1}{2}}$, hence by the theorems of Rellich and Arzela-Ascoli, some subsequence of $u_{j}$ converges uniformly in the $L^{2}$ norm on each finite time interval to $u(t)$, in the sense that

$$
\lim _{n \rightarrow \infty}\left\|u_{j(n)}-u\right\|_{C\left([-T, T], L^{2}\right)}=0 \text { for all } T<\infty .
$$

It follows that $u \in L^{\infty} H^{1}$, and thus by interpolation that for all $s<1$,

$$
\lim _{n \rightarrow \infty}\left\|u_{j(n)}-u\right\|_{C\left([-T, T], H^{s}\right)}=0 \text { for all } T<\infty .
$$

By Sobolev embedding we deduce that $F_{j}\left(u_{j}\right) \rightarrow F(u)$ in $C\left([-T, T], L^{2}\right)$, hence $u$ is the solution to (59), unique by above. The conservation of mass in (66) follows by uniform convergence in the $L^{2}$ norm and conservation of mass for $u_{j}$. To conclude, we observe that by energy conservation for $u_{j}$ and Fatou's lemma, for each $t_{1}$ we have

$$
\int_{\Omega}\left|d_{x} u\left(t_{1}\right)\right|_{\mathrm{g}}^{2}+G\left(\left|u\left(t_{1}\right)\right|^{2}\right) \leq \int_{\Omega}\left|d_{x} u\left(t_{0}\right)\right|_{\mathrm{g}}^{2}+G\left(\left|u\left(t_{0}\right)\right|^{2}\right) .
$$

On the other hand, $u$ is the unique solution with data $u\left(t_{1}\right)$ at time $t_{1}$, and the inequality is thus symmetric under exchange of $t_{0}$ and $t_{1}$.

It remains to prove existence of energy conserving solutions to (59) for $H^{1}$ data, in case $G(r) \in C_{c}^{\infty}(\mathbb{R})$. For convenience set $t_{0}=0$. We introduce $w(t)=$ $\exp (-i t \Delta) u(t)$, and write $(59)$ as

$$
w(t)=u_{0}-i \int_{0}^{t} e^{-i s \Delta} F\left(e^{i s \Delta} w(s)\right) d s .
$$

Since $F(z)=G^{\prime}\left(|z|^{2}\right) z \in C_{c}^{\infty}(\mathbb{C})$, the map $u \rightarrow F(u)$ is globally Lipschitz on $L^{2}(\Omega)$, and one has existence, uniqueness, and Lipschitz dependence on initial data for $C^{1} L^{2}$ solutions of (68), given by the limit of $w_{n}(t)$, where $w_{0}(t)=u_{0}$, and

$$
w_{n+1}(t)=u_{0}-i \int_{0}^{t} e^{-i s \Delta} F\left(e^{i s \Delta} w_{n}(s)\right) d s .
$$

Convergence of $w_{n}$ to $w$ is uniform in the $L^{2}$ norm on any compact interval. From unitarity of $\exp (i t \Delta)$ on $H^{k}$ (with norm defined spectrally), and the bound

$$
\|F(w(s))\|_{H^{1}} \leq K\|w(s)\|_{H^{1}},
$$

one sees from (69) and weak limits, and using (68) to express $w^{\prime}(t)$, that

$$
\|w(t)\|_{H^{1}} \leq\left\|u_{0}\right\|_{H^{1}} \exp (K t), \quad\left\|w^{\prime}(t)\right\|_{H^{1}} \leq K\left\|u_{0}\right\|_{H^{1}} \exp (K t) .
$$

It remains to prove the conservation laws (66) on an interval $[0, T]$, for a $T$ depending only on $\left\|u_{0}\right\|_{H^{1}}$ and $F$; uniqueness yields global conservation. To do this, we will prove for such a $T$ that if $u_{0} \in H^{2}$, then $w \in C^{1}\left([0, T], H^{2}\right)$, hence $u \in$ $C^{1}\left([0, T], L^{2}\right)$. Together this is sufficient regularity to see that $(66)$ holds on $[0, T]$ 
for $u_{0} \in H^{2}$. Density and Fatou's lemma yields mass conservation and (67) for $H^{1}$ data; uniqueness then yields (66).

We start by noting that

$$
\|F(u(s))\|_{H^{2}} \lesssim\|u(s)\|_{W^{1,4}}^{2}+\|u(s)\|_{H^{2}} \lesssim\|u(s)\|_{H^{2}}^{2}+\|u(s)\|_{H^{2}} .
$$

Iterating (69) yields $\|u\|_{L^{\infty}\left(\left[0, T^{\prime}\right], H^{2}\right)} \leq 2\left\|u_{0}\right\|_{H^{2}}$ for some $T^{\prime}>0$ depending on $\left\|u_{0}\right\|_{H^{2}}$. It suffices then to prove, for some $C$ and $T$ depending only on $\left\|u_{0}\right\|_{H^{1}}$, that if $T^{\prime} \leq T$ and $\|u\|_{L^{\infty}\left(\left[0, T^{\prime}\right], H^{2}\right)}<\infty$, then $\|u\|_{L^{\infty}\left(\left[0, T^{\prime}\right], H^{2}\right)} \leq C\left\|u_{0}\right\|_{H^{2}}$. Theorem 1.2 and (59) yield

$$
\begin{aligned}
\|u\|_{L^{4}\left(\left[0, T^{\prime}\right], W^{1,4}\right)}^{2} & \lesssim\left\|u_{0}\right\|_{H^{3 / 2}}^{2}+\left(\int_{0}^{T^{\prime}}\|F(u(s))\|_{H^{3 / 2}} d s\right)^{2} \\
& \lesssim\left\|u_{0}\right\|_{H^{1}}\left\|u_{0}\right\|_{H^{2}}+\int_{0}^{T^{\prime}}\|F(u(s))\|_{H^{1}}\|F(u(s))\|_{H^{2}} d s,
\end{aligned}
$$

and we can also use (59) to bound $\|u\|_{L_{T^{\prime}}^{\infty} H^{2}} \leq\left\|u_{0}\right\|_{H^{2}}+\|F(u)\|_{L_{T^{\prime}}^{1} H^{2}}$. By the bounds (70), we combine these estimates, assuming $T^{\prime} \leq T \leq 1$, to yield

$$
\|u\|_{L_{T^{\prime}}^{\infty} H^{2}}+\|F(u)\|_{L_{T^{\prime}}^{2} H^{2}} \leq C\left\|u_{0}\right\|_{H^{2}}+C T^{\frac{1}{2}}\|u\|_{L_{T^{\prime}}^{\infty} H^{2}}+C T^{\frac{1}{2}}\|F(u)\|_{L_{T^{\prime}}^{2} H^{2}}
$$

where $C \lesssim\left\|u_{0}\right\|_{H^{1}}$. Taking $T$ small yields the desired result.

We conclude by noting that the above argument shows that $u \in C\left([0, T], H^{2}\right)$ for all finite $T$ if $u_{0} \in H^{2}$, but possibly with exponential growth of the $H^{2}$ norm, with the growth constant depending on $\left\|u_{0}\right\|_{H^{1}}$.

Acknowledgements. The authors would like to thank the referee for suggesting the application of our methods to semilinear Schrödinger equations on compact manifolds in Section 6.

\section{REFERENCES}

[1] Anton, R.: Global existence for defocusing cubic NLS and Gross-Pitaevskii equations in exterior domains. J. Math. Pures Appl. 89(4), 335-354 (2008)

[2] Blair, M.D., Smith, H.F., and Sogge, C.D.: On Strichartz Estimates for Schrödinger Operators in Compact Manifolds with Boundary. Proc. Amer. Math. Soc. 136, 247-256 (2008)

[3] Blair, M.D., Smith, H.F., and Sogge, C.D.: Strichartz estimates for the wave equation on manifolds with boundary. Ann. Inst. H. Poincaré Anal. Non Linéaire 26(5), 1817-1829 (2009)

[4] Burq, N., Gérard, P., and Tzvetkov, N.: On nonlinear Schrodinger equations in exterior domains. Ann. Inst. H. Poincaré Anal. Non Linéaire 21(3), 295-318 (2004)

[5] Burq, N., Gérard, P., and Tzvetkov, N.: Strichartz inequalities and the nonlinear Schrödinger equation on compact manifolds. Amer. J. Math. 126, 569-605 (2004)

[6] Constantin, P. and Saut, J.C.: Local smoothing properties of dispersive equations. J. Amer. Math Soc. 1, 413-439 (1988)

[7] Davies, E.B.: Heat Kernels and Spectral Theory. Cambridge University Press, Cambridge, 1990.

[8] Ginibre, J. and Velo, G.: On the global Cauchy problem for some nonlinear Schrödinger equations. Ann. Inst. H. Poincaré Anal. Non Linéaire 1(4), 309-323 (1984)

[9] Herr, S.: The quintic nonlinear Schrödinger equation on three-dimensional Zoll manifolds. To appear in Amer. J. Math.

[10] Herr, S., Tataru, D., and Tzvetkov, N.: Global well-posedness of the energy critical Nonlinear Schrödinger equation with small initial data in $H^{1}\left(T^{3}\right)$. Duke Math. J. 159(2), 329-349 (2011)

[11] Ionescu, A.D. and Pausader, B.: The energy-critical defocusing NLS on $T^{3}$. To appear in Duke Math. J.

[12] Ivanovici, O.: On the Schrödinger equation outside strictly convex obstacles. Anal. PDE 3(3), 261-293 (2010) 
[13] Ivanovici, O. and Planchon, F.: On the energy critical Schrödinger equation in 3D nontrapping domains. Ann. Inst. H. Poincaré Anal. Non Linéaire 27(5), 1153-1177 (2010)

[14] Journé, J.L., Soffer, A., and Sogge, C.D.: Decay estimates for Schrödinger operators. Comm. Pure Appl. Math. 44(5), 573-604 (1991)

[15] Keel, M. and Tao, T.: Endpoint Strichartz Estimates. Amer. J. Math. 120, 955-980 (1998)

[16] Koch, H. and Tataru, D.: Dispersive estimates for principally normal operators. Comm. Pure Appl. Math. 58, 217-284 (2005)

[17] Planchon, F. and Vega, L.: Bilinear Virial Identities and Applications. Ann. Sci. Éc. Norm. Supér. (4) 42(2), 261-290 (2009)

[18] Sjölin, P.: Regularity of solutions to Schrödinger equations. Duke Math J. 55, 699-715 (1987)

[19] Staffilani, G. and Tataru, D.: Strichartz estimates for a Schrödinger operator with nonsmooth coefficients. Comm. Partial Differential Equations 27(7-8), 1337-1372 (2002)

[20] Smith, H.F. and Sogge, C.D.: On the $L^{p}$ norm of spectral clusters for compact manifolds with boundary. Acta Math. 198, 107-153 (2007)

[21] Strichartz, R.: Restriction of Fourier transform to quadratic surfaces and decay of solutions to the wave equation. Duke Math. J. 44(3), 705-714 (1977)

[22] Taylor, M.: Pseudodifferential Operators and Nonlinear PDE. Progress in Mathematics, vol. 100, Birkhäuser, Boston, 1991.

[23] Vega, L.: Schrödinger equations: pointwise convergence to the initial data. Proc. Amer. Math Soc 102, 874-878 (1988) 